\title{
Tumor-conditional anti-CTLA4 uncouples antitumor efficacy from immunotherapy-related toxicity
}

\author{
Chien-Chun Steven Pai, ${ }^{1}$ Donald M. Simons, ${ }^{2}$ Xiaoqing Lu, ${ }^{2}$ Michael Evans, ${ }^{3}$ Junnian Wei, ${ }^{3}$ Yung-hua Wang, ${ }^{3}$ Mingyi Chen, ${ }^{4}$ \\ John Huang, ${ }^{1}$ Chanhyuk Park, ${ }^{1}$ Anthony Chang, ${ }^{1}$ Jiaxi Wang, ${ }^{1}$ Susan Westmoreland, ${ }^{2}$ Christine Beam, ${ }^{2}$ Dave Banach, ${ }^{2}$ \\ Diana Bowley, ${ }^{2}$ Feng Dong, ${ }^{2}$ Jane Seagal, ${ }^{2}$ Wendy Ritacco, ${ }^{2}$ Paul L. Richardson, ${ }^{5}$ Soumya Mitra, ${ }^{2}$ Grace Lynch, ${ }^{2}$ \\ Pete Bousquet, ${ }^{2}$ John Mankovich, ${ }^{5}$ Gillian Kingsbury, ${ }^{2}$ and Lawrence Fong ${ }^{1,6}$ \\ 1Department of Hematology and Oncology, School of Medicine, UCSF, San Francisco, California, USA. ${ }^{2}$ AbbVie Bioresearch Center, Worcester, Massachusetts, USA. ${ }^{3}$ Department of Radiology and Biomedical \\ Imaging, School of Medicine, UCSF, San Francisco, California, USA. ${ }^{4}$ Department of Hematopathology, School of Medicine, University of Texas Southwestern Medical Center, Dallas, Texas, USA. ${ }^{5}$ AbbVie Inc., \\ North Chicago, Illinois, USA. ${ }^{6}$ Parker Immunotherapy Institute, Helen Diller Family Comprehensive Cancer Center, UCSF, San Francisco, California, USA
}

\begin{abstract}
While immune checkpoint blockade leads to potent antitumor efficacy, it also leads to immune-related adverse events in cancer patients. These toxicities stem from systemic immune activation resulting in inflammation of multiple organs, including the gastrointestinal tract, lung, and endocrine organs. We developed a dual variable domain immunoglobulin of anti-CTLA4 antibody (anti-CTLA4 DVD, where CTLA4 is defined as cytotoxic T lymphocyte-associated antigen-4) possessing an outer tumor-specific antigen-binding site engineered to shield the inner anti-CTLA4-binding domain. Upon reaching the tumor, the outer domain was cleaved by membrane type-serine protease 1 (MT-SP1) present in the tumor microenvironment, leading to enhanced localization of CTLA4 blockade. Anti-CTLA4 DVD markedly reduced multiorgan immune toxicity by preserving tissue-resident Tregs in Rag 1/-- mice that received naive donor CD4+ T cells from WT C57BL/6j mice. Moreover, anti-CTLA4 DVD induced potent antitumor effects by decreasing tumor-infiltrating Tregs and increasing the infiltration of antigen-specific CD8+ $T$ lymphocytes in TRAMP-C2-bearing C57BL/6j mice. Treg depletion was mediated through the antibody-dependent cellular cytotoxicity (ADCC) mechanism, as anti-CTLA4 without the Fc $\gamma$ R-binding portion (antiCTLA4 DANA) spared Tregs, preventing treatment-induced toxicities. In summary, our results demonstrate an approach to anti-CTLA4 blockade that depletes tumor-infiltrating, but not tissue-resident, Tregs, preserving antitumor effects while minimizing toxicity. Thus, our tumor-conditional anti-CTLA4 DVD provides an avenue for uncoupling antitumor efficacy from immunotherapy-induced toxicities.
\end{abstract}

\section{Introduction}

Immune checkpoint inhibition (CPI) has demonstrated promising results in both preclinical models $(1,2)$ and in the clinic (3). Yet several issues remain and severely hinder the clinical benefits, including the fact that only a small proportion of patients respond to these therapies, while some others might develop treatment-associated organ toxicities (4).Of the types of CPI, cytotoxic T lymphocyteassociated antigen-4 (CTLA4) demonstrated potent antitumor effects and has been approved by the FDA as a therapy in advanced melanoma patients. Despite the promising clinical outcomes, in a

\section{Related Commentary: p. 75}

Authorship note: CCSP and DMS contributed equally to this work. GK and LF contributed equally to this work.

Conflict of interest: LF received research funding from AbbVie, Merck, Roche-Cenentech, and Bristol-Myers Squibb Inc. DMS, XL, SW, CB, D Banach, D Bowley, FD, JS, WR, PLR, SM, GL, and PB are research scientists at AbbVie Bioresearch Center. DMS, JM, and CK are former research scientists at AbbVie Bioresearch Center. License: Copyright 2019, American Society for Clinical Investigation.

Submitted: July 24, 2018; Accepted: October 24, 2018.

Reference information: J Clin Invest. 2019;129(1):349-363.

https://doi.org/10.1172/JCl123391. recent phase III trial, $28 \%$ of melanoma patients receiving ipilimumab (anti-CTLA4) developed grade 3 and grade 4 immune-related adverse events (irAEs) presenting as multiorgan toxicities (5). Therefore, there exists an urgent need to develop strategies that can reduce toxicity while maintaining the antitumor effects from CPI.

Several mechanisms have been linked to CTLA4 blockademediated toxicities. Blocking of CTLA4 signaling with an antagonist can potentially activate $\mathrm{T}$ cell clones that react to self-antigens, resulting in autoimmune disease-like symptoms (6-8). In addition to conventional T cells, Tregs also express high amounts of CTLA4 (9). The binding of blocking Abs to CTLA4 can potentially deplete Tregs through antibody-dependent cellular cytotoxicity (ADCC) (10). Systemic depletion of tissue-resident Tregs impedes peripheral tolerance, making patients more prone to developing irAEs after anti-CTLA4 treatments. Therefore, we hypothesized that developing a strategy to limit systemic exposure of CTLA4 blockade while depleting tumor-infiltrating Tregs can potentially maintain antitumor activity without the risk of irAEs.

To achieve this, we engineered a class of anti-CTLA4 Ab that is preferentially released within the tumor microenvironment, which we named CTLA4 dual variable domain Ig (anti-CTLA4 DVD). The anti-CTLA4 DVD is designed to have the inner CTLA4-binding 
A

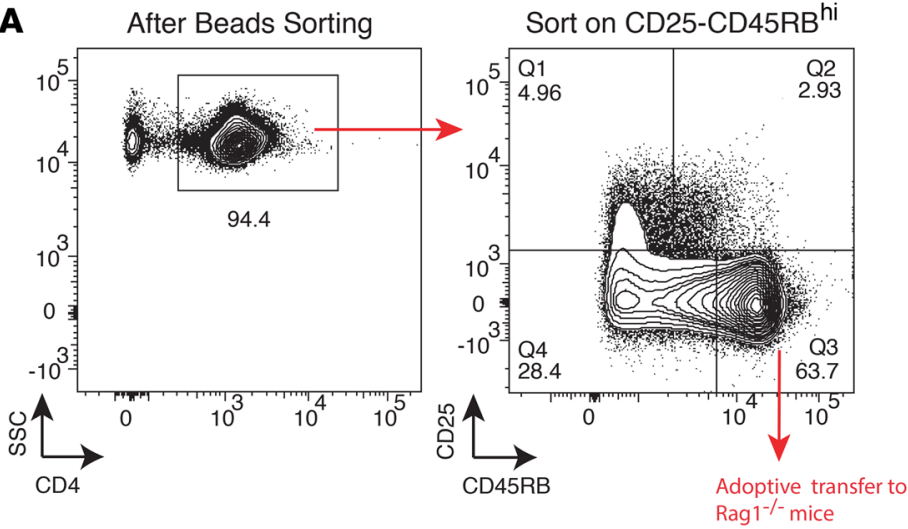

C
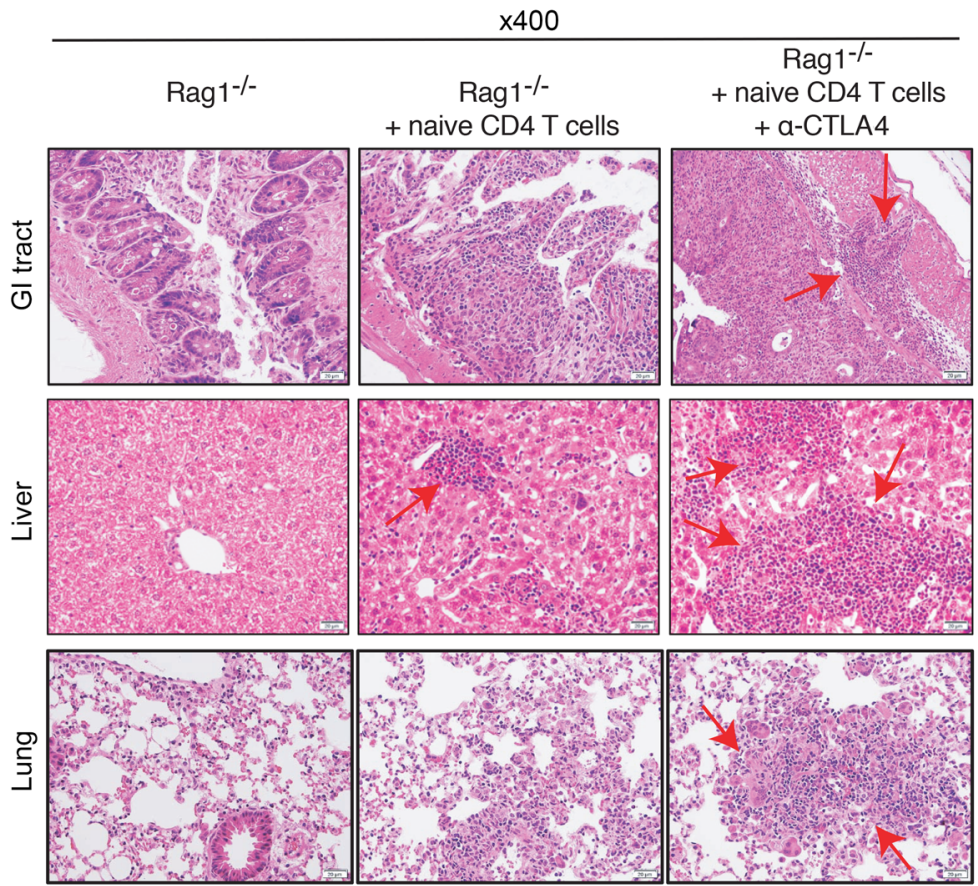

$\mathbf{F}$

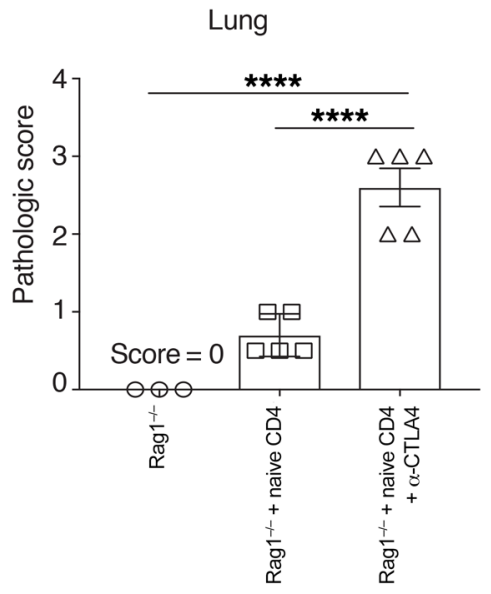

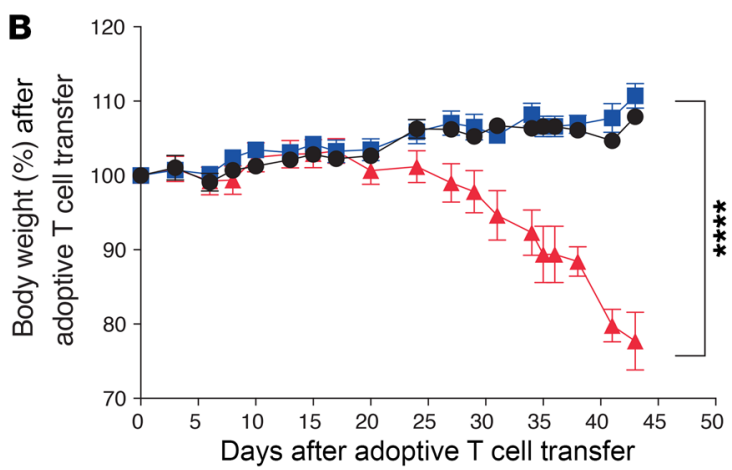

Rag $1 \%$

- Rag $1 \%$ + naive CD4 T cells

t Rag $1{ }^{-/}+$naive CD4 T cells + a-CTLA4
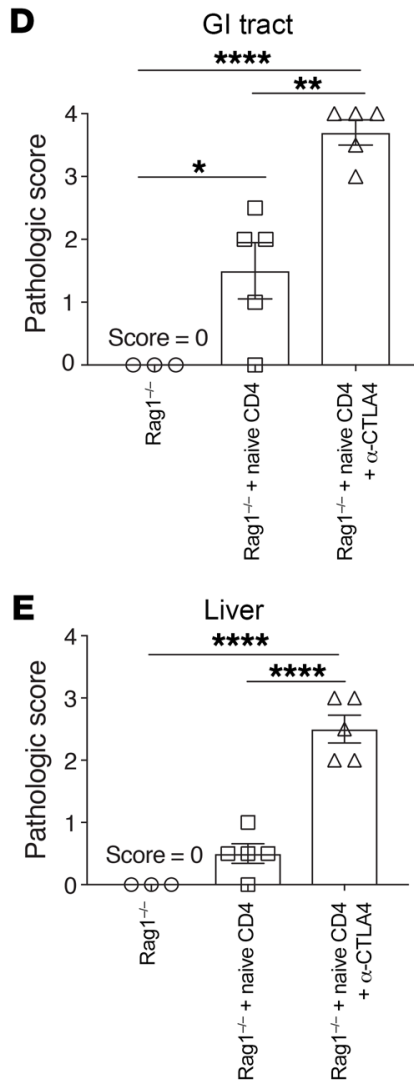

G Isotype control H

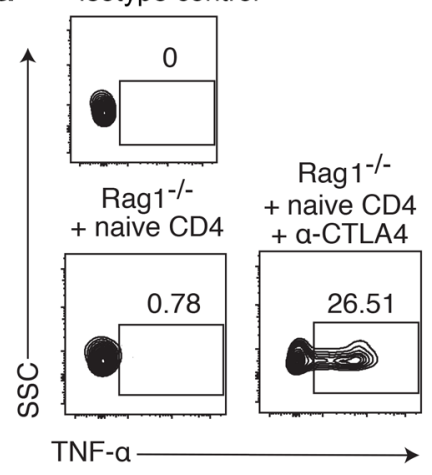

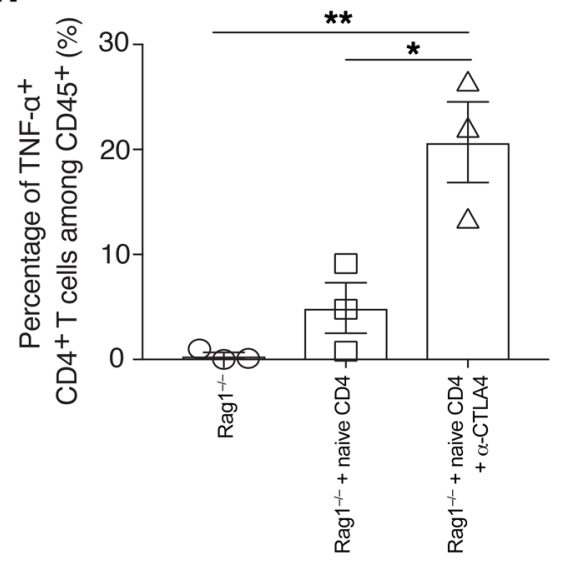


Figure 1. Anti-CTLA4-mediated immune-related toxicities in a murine model. Eight- to ten-week-old male Rag $1^{1-1-}$ mice (on C57BL/6j background) were adoptively transferred with purified CD4+CD25-CD45RB hi cells from WT mice. (A) Sorting strategy. (B) Body weight loss over time after treatment. (C) Different organs were harvested at day 45 , and pathological changes were examined using $\mathrm{H} \& \mathrm{E}$ staining under a microscope. Arrows indicate lymphocytic infiltration and pathological changes. (D-F) Target organ pathological scores were evaluated by a board-certified pathologist in a single-blind fashion. (C) Splenocytes were harvested at day 45 from different treatment groups, and $\mathrm{CD} 4^{+} \mathrm{T}$ cells were examined for TNF- $\alpha$ secretion by flow cytometry. (H) Percentage of TNF- $\alpha^{+} C D 4^{+} T$ cells among CD45+ cells. Experiments were conducted twice, and data were shown with 5 mice per group. Three mice from the Rag ${ }^{1-1-}$ only group were used as negative control. For the TNF- $\alpha$ experiment, data were collected from 3 mice per group. Bars represent mean \pm SEM. ${ }^{*} P<0.05 ;{ }^{* *} P<0.01$; ${ }^{* * *} P<0.0001$, 1-way ANOVA or 2-way ANOVA with post hoc Tukey's test.

domain shielded by an outer tumor-targeting anti-prostate stem cell antigen (PSCA) domain. Activated membrane type-serine protease 1 (MT-SP1) found within the tumor microenvironment can cleave this shield, exposing the inner CTLA4-binding site. We found that this targeted release of anti-CTLA4 within tumors can deplete tumor Tregs, but spares tissue-resident Tregs, allowing the preservation of antitumor activities. Our findings demonstrate what we believe is a new class of tumor-conditional CTLA4 Ab and a platform that can potentially be applied in the clinic.

\section{Results}

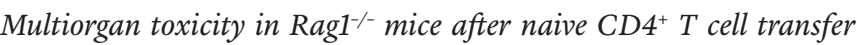
and CTLA4 blockade. Cancer patients receiving ipilimumab (antiCTLA4) develop severe treatment-associated toxicities, including colitis and hepatic or lung inflammatory response, resulting in the cessation of treatment. In contrast, tumor-bearing mice receiving anti-CTLA4 do not develop toxicities, as seen in the clinic, despite multiple doses (data not shown). It has been shown that patients who developed irAEs after anti-CTLA4 treatment tended to show expansion of autoreactive $\mathrm{CD} 4^{+} \mathrm{T}$ cells (11). Therefore, to mimic CD4-mediated toxicity by CTLA4 blockade, we engaged in adoptive transfer of naive $\mathrm{CD} 4^{+} \mathrm{T}$ cells $\left(\mathrm{CD} 4^{+} \mathrm{CD} 45 \mathrm{RB}^{\mathrm{hi}} \mathrm{CD} 25^{-}\right)$from WT donor mice into $\operatorname{Rag}^{-/-}$recipient mice (Figure $\left.1 \mathrm{~A}\right)$. Similar to the symptoms observed in clinic, mice that received CTLA 4 blockade presented with diarrhea and significant weight loss compared with mice without anti-CTLA4 treatment (Figure 1B; $P<0.0001$ ). The anti-CTLA4 treatment induced typical irAEs that involved multiple organs (gastrointestinal [GI] tract, liver, and lung). Specifically, inflammatory changes consistent with irAEs were observed, including colitis, hepatitis, and pneumonitis (Figure 1C). In the GI tract, a dense lymphocytic infiltrate was seen in the colonic mucosa, with associated architectural changes characterized by marked epithelial damage, crypt distortion, surface erosion, and transmural inflammation (Figure 1C). In the liver, marked portal inflammation, with interface hepatitis, patchy steatosis, and cholestasis, was observed (Figure 1C). In the lung, marked peribronchial and perivascular lymphocytic infiltrate and focal alveolitis were found (Figure 1C). The anti-CTLA4-treated group also demonstrated significantly higher pathology scores compared with either the $\mathrm{Rag1}^{-/-}$control group or the naive $\mathrm{CD}^{+} \mathrm{T}$ cell-transferred group, illustrating the toxic effects mediated through CTLA4 blockade treatments (Figure 1, D-F). It has also been shown that patients who develop irAEs after anti-CTLA4 administration possessed elevated TNF- $\alpha$ levels (12). Likewise, we observed a similar pattern in our model in which mice receiving anti-CTLA4 treatments had significantly increased TNF- $\alpha$ production from donor $\mathrm{CD} 4^{+} \mathrm{T}$ cells (Figure 1, G and $\mathrm{H}$ ). Overall, our data indicate that adoptive transfer of $\mathrm{CD}^{+} \mathrm{T}$ cells from WT mice into recipient Rag1/- mice can closely mimic the characteristics of anti-CTLA4-mediated multiorgan toxicities seen in the clinic.

Engineering a tumor-conditional anti-CTLA4 Ab. Systemic administration of CTLA4 blockade can potentially activate autoreactive $\mathrm{T}$ cell clones that target self-antigens and result in multiorgan toxicities (8). Previously, it has been shown that limiting the effects of CTLA4 blockade within the tumor microenvironment can potentially reduce such toxicities (13). We sought to build an engineered CTLA4 blockade that maintains antitumor activity, but also to limit its systemic exposure. To achieve that, we created DVD Ig, which is a tetravalent bispecific Ab-like molecule containing an Fc region and 2 pairs of variable domains joined in tandem by a short flexible linker (Figure 2A). In some DVDs, the outer variable domain (OD) could potentially interfere with ligand binding of the inner variable domains (ID) through steric hindrance, so we sought to solve this problem with a cleavable linker. We proposed an ID-OD linker that could be cleaved by proteases within the tumor microenvironment, acting as a switch to expose the ID from the OD for ligand binding. We hypothesized that a conditional DVD composed of ODs specific for a tumor antigen together with a linker that is cleavable by proteases found in the tumor could be used to reduce the systemic toxicity associated with CTLA4-neutralizing Abs.

To test this hypothesis, we designed a tumor-targeting conditional DVD that could be used to treat the TRAMP-C2 prostate cancer model in mice. We have previously shown that established $\left(150 \mathrm{~mm}^{3}\right)$ TRAMP-C2 tumors are rejected following treatment with the CTLA4-neutralizing Ab (14). Therefore, we chose the variable domains from the CTLA4-blocking Ab to serve as the IDs for the tumor-targeted conditional DVD. Additionally, TRAMP tumors express high levels of the murine homologue of the tumor antigen PSCA, so a mouse PSCA-specific mAb (10E8 clone) was generated to target the tumor as the OD of the conditional DVD (herein referred to as anti-CTLA4 DVD) (Figure 2A). Furthermore, TRAMP-C2 tumors expressed high levels of the MT-SP1 (Figure 2B) (15). Therefore, a DVD linker containing the sequence LSGRSDNH should be susceptible to cleavage by MT-SP1. To determine whether this linker sequence could be used to activate anti-CTLA4 DVD at the TRAMP-C2 tumor, we designed a peptide probe comprising Oregon Green conjugated to the N-terminal of the linker peptide with a fluorescent quencher conjugated to the C-terminal residue (Figure 2C). Due to Förster resonance energy transfer-based (FRET-based) quenching, the Oregon Green dye would only fluoresce if it was separated from the quencher after cleavage of the peptide. Incubation of the peptide probe with recombinant MT-SP1 resulted in a dose-dependent increase in fluorescence (Figure 2D). We also observed an increase in fluorescent signal when this probe was incubated with whole cell lysate from TRAMP-C2 tumor cells or when the probe was incubated on (nonfixed) cryosections of TRAMP-C2 tumors at $37^{\circ} \mathrm{C}$ (Fig- 
A $\operatorname{mAb} \# 1(\mathrm{a}-\mathrm{CTLA}-4)$

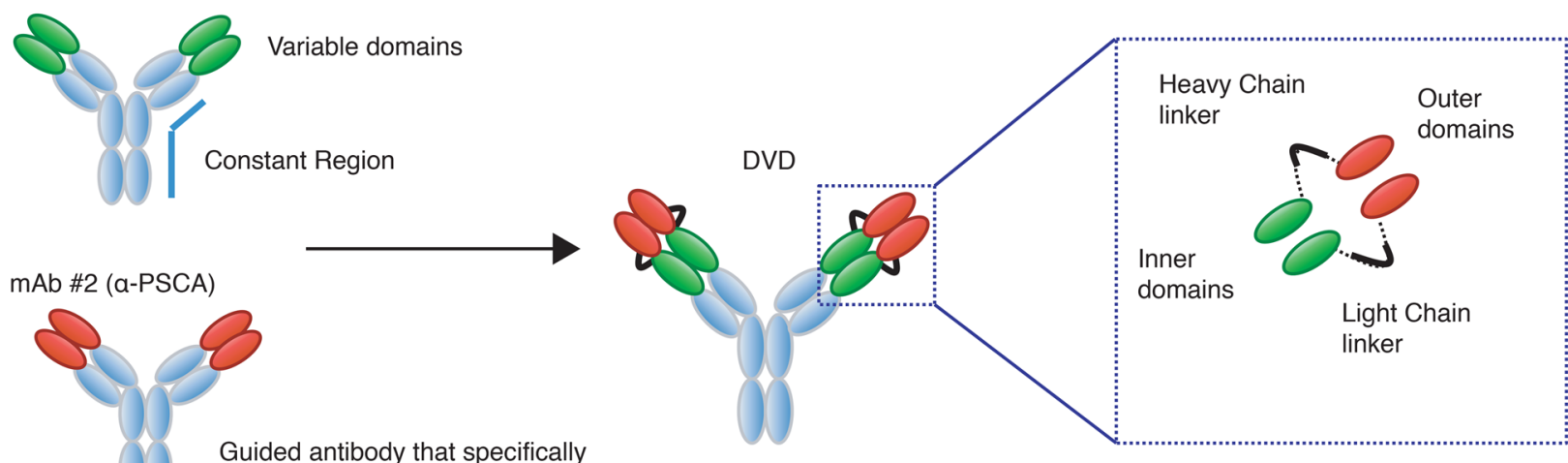

targets cancer cells

B

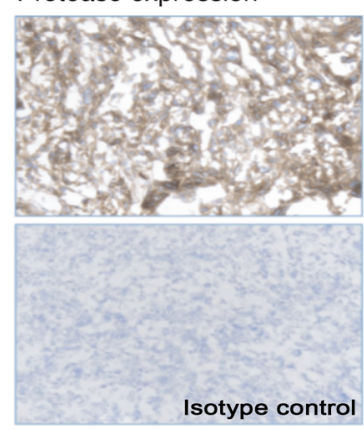

C

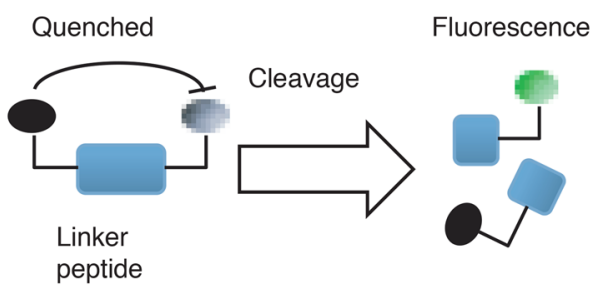

D

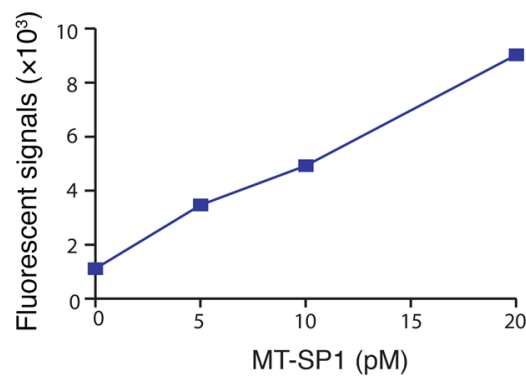

$\mathbf{F}$ Cleavage of TRAMP-C2
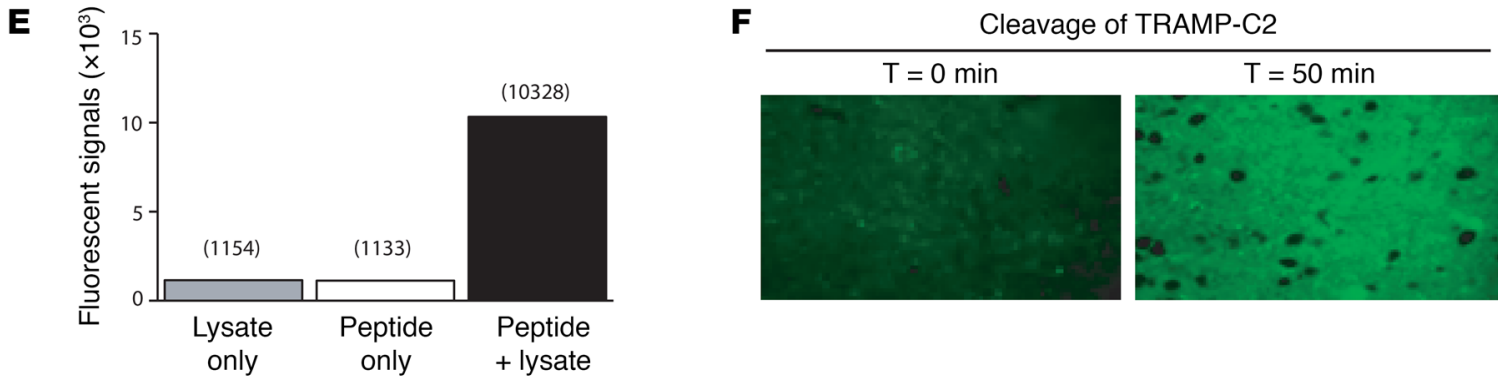

Figure 2. Engineered CTLA4 blockade with bispecific DVD Ig design. (A) Diagram illustrating DVD technology. The anti-CTLA4 DVD is composed of 1 outer domain (antigen-specific binding site) and 1 inner domain (CTLA4-binding site). The outer domain and inner domain are connected by cleavable linkers. (B) Immunohistochemistry of MT-SP1 expression in a TRAMP-C2 tumor section. Original magnification: $\times 200$. (C) Illustration of the fluorescent peptide probes used to evaluate the cleavage of linker peptides. (D) Fluorescent peptide probes containing the conditional DVD cleavable linker sequence were incubated with recombinant MT-SP1 to demonstrate cleavage under different concentrations. Cleavage was also achieved through cocultures of probes with TRAMP-C2 tumor lysate (E) and in tumor sections (F). Representative figures from each in vitro experiment are shown. Each in vitro experiment was conducted 3 times independently. Original magnification: $\times 400$.

ure 2, $\mathrm{E}$ and F). Together, these data indicated that a DVD linker designed to contain a MT-SP1 cleavage site within its sequence can be cleaved by proteases found in TRAMP-C2 tumors and that this linker sequence can be used as the basis for designing the anti-CTLA4 DVD.

Identification and characterization of a conditional PSCA/CTLA4 $D V D$. Candidate anti-CTLA4 DVD screens were conducted to identify the variable domain and linker combinations with reduced ID binding. We subsequently identified a candidate DVD that bound to PSCA-overexpressing cells with the same affinity as the 10E8 clone $\mathrm{mAb}$. The shielded $\mathrm{Ab}$ also had a significant reduction in binding to CTLA4-overexpressing cells and did not inhibit the binding of B7-2 to CTLA4-overexpressing cell lines (Supplemental Figure 1, A-D; supplemental material available online with this article; https:// doi.org/10.1172/JCI123391DS1). However, we did not observe any cleavage of the linkers on this DVD when it was incubated with recombinant MT-SP1 (data not shown). The heavy chain and light chain linkers used in this DVD were only 6 and 5 amino acids long, respectively. Therefore, we hypothesized that extending the linkers could improve cleavage by making the linkers more accessible to the protease. To evaluate this hypothesis, we made a series of PSCA/ CTLA4 DVDs (10E8/24H2) with extended heavy chain (HC) and light chain (LC) linker sequences (Figure 3A). Incubation of these DVDs with recombinant MT-SP1 showed that the length of the light 
A

Linker optimization strategy for cleavage
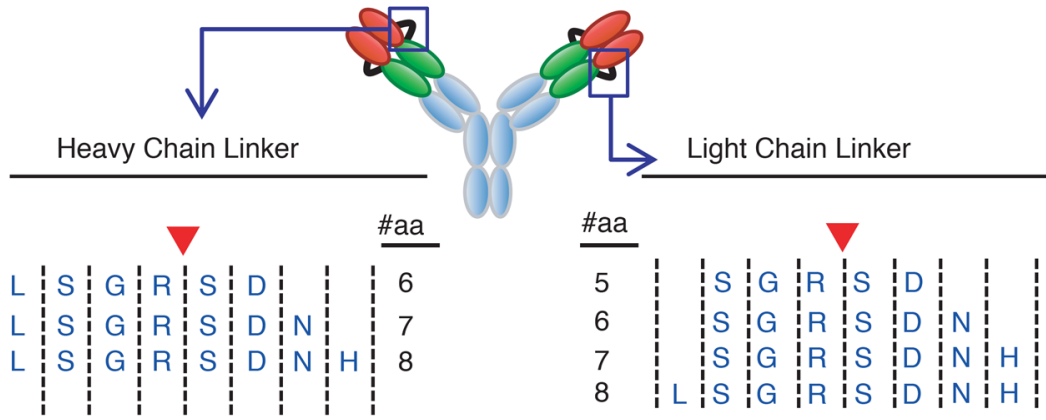

aa

6

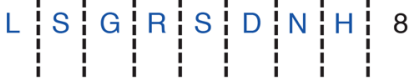

B

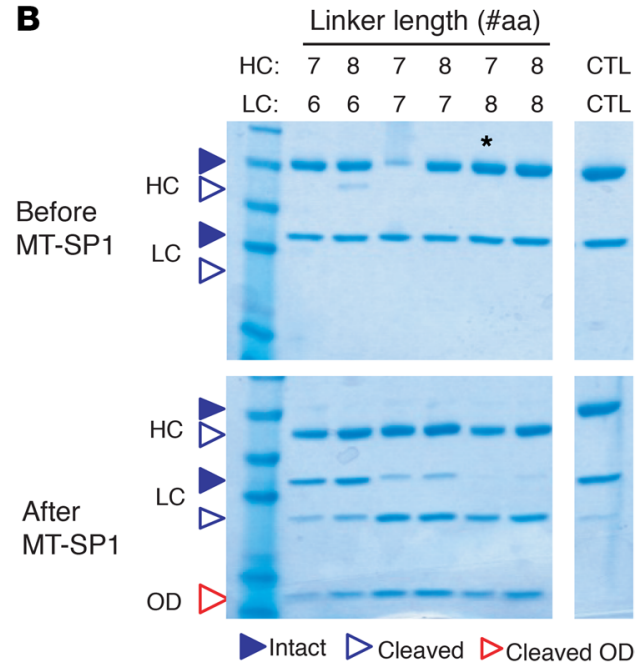

E

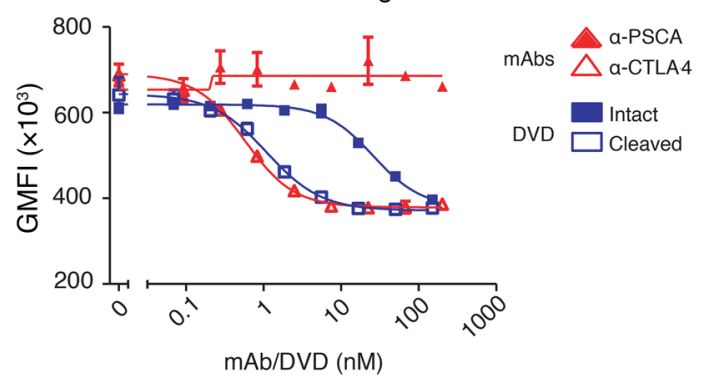

G

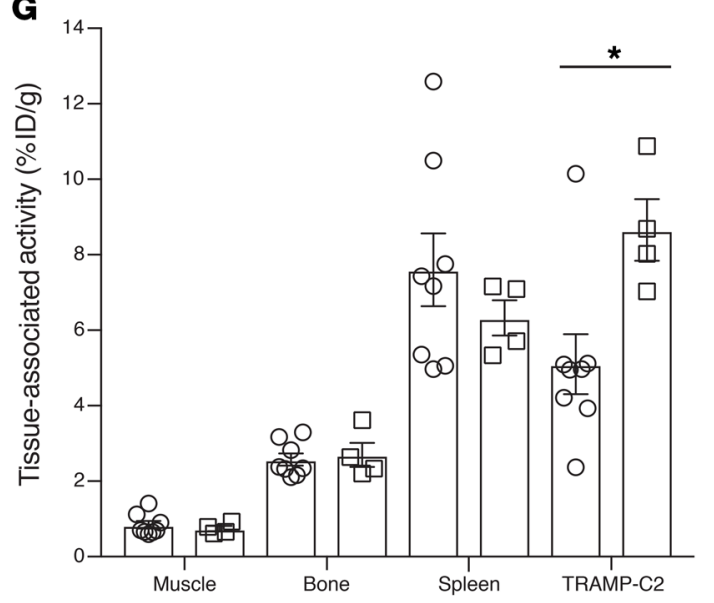

Figure 3. Protease-cleavable linkers can act as a switch to convert the specificity of a DVD from a tumor antigen to a therapeutic target. A panel of DVDs was made to identify the optimal linker for conditional activation. All DVDs had identical ODs (anti-PSCA) and IDs (anti-CTLA4), but used different linkers. (A) Schematic showing the linker sequences used to optimize cleavage by MT-SP1. (B) Reducing SDS-PACE of DVDs before and after incubation with MT-SP1. CTL indicates DVD made with linkers lacking the MT-SP1 cleavage site (LSGSDN/SCSDN). Asterisk indicates candidate DVD (5037) with optimal linker lengths that was advanced for further testing. (C and D) After incubation with MT-SP1, intact DVDs or cleaved DVDs were incubated with HEK293 cells overexpressing PSCA (C) or CTLA4 (D) and specific bindings were detected by secondary labeling and FACS. (E) Intact or cleaved DVDs were incubated with HEK293 cells overexpressing CTLA4 in the presence of CD86, and specific binding of CD86 to the cells was detected by flow cytometry. Each in vitro experiment was conducted 3 times independently. A representative figure from each in vitro experiment is shown. (F) Mice were implanted with s.c. TRAMP-C2 tumors and injected with either anti-CTLA4 or anti-CTLA4 DVD. The representative figure from each treatment arm demonstrates the biodistribution of anti-CTLA4 and anti-CTLA4 DVD at 48 hours by PET/CT. The upper portion is the transverse plane of the CT scan. The highlight portion is the tumor. The lower panel is the coronal plane of the CT scan. (C) Quantification of PET/CT tissue distribution of anti-CTLA4 and anti-CTLA4 DVD at 48 hours. Data were conducted with 2 independent experiments. Each treatment arm was collected from 8 mice per group for the anti-CTLA4-treated group and 4 mice per group for the anti-CTLA4 DVD-treated group. Bars represent mean \pm SEM. ${ }^{*} P<0.05,2$ tailed Student's $t$ test. 
A

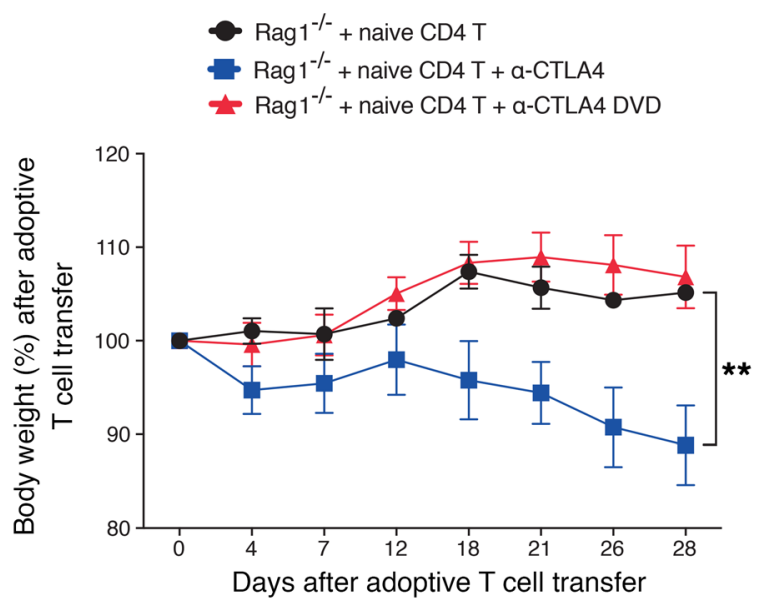

B Rag $^{1--}$ + naive CD4
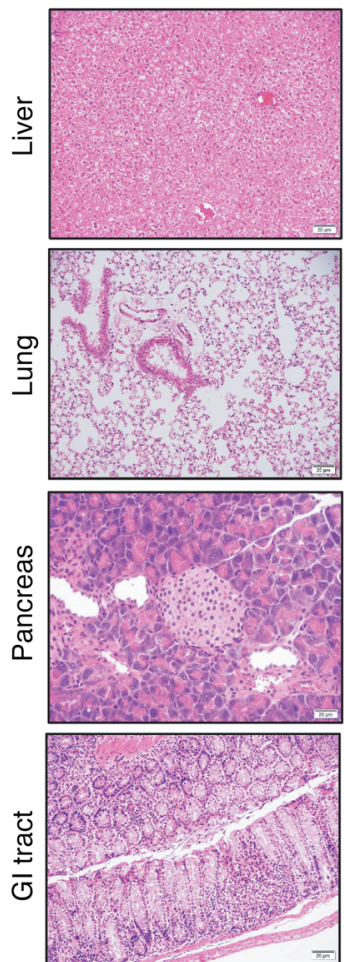

Rag $1^{-/}+$naive CD4 + a-CTLA4
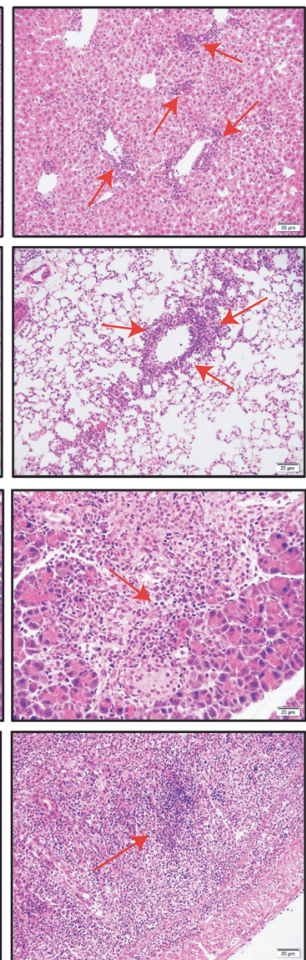

Rag $1 \%$ + naive CD4 + a-CTLA4 DVD

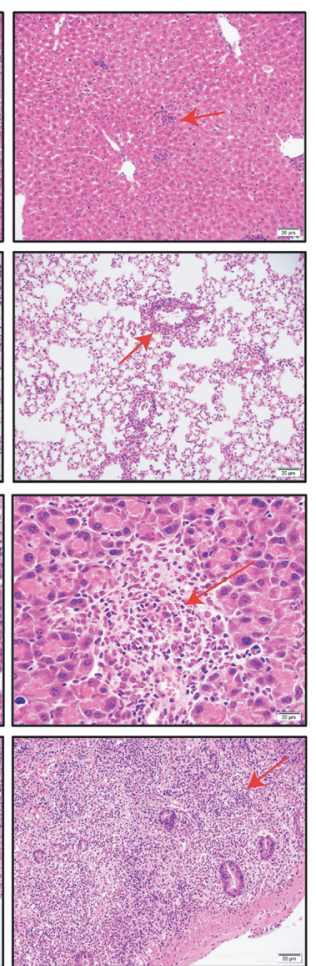

Figure 4. Anti-CTLA4 DVD reduced treatment-associated toxicity in mice. Eight- to ten-week-old male Rag1-/- mice (on a C57BL/6j background) were adoptively transferred with purified CD4 ${ }^{+} \mathrm{CD} 25-{ }^{-} \mathrm{CD} 45 \mathrm{RB}^{\mathrm{hi}}$ cells from WT mice. Recipient mice were then treated with either anti-CTLA4, anti-CTLA4 DVD, or isotype control. (A) Percentage of body weight lost among different treatment groups. (B) Mice were harvested at day 28 , and multiorgan pathology was examined among different treatment groups. Arrows represent lymphocytic infiltration in the tissues. Experiments were conducted twice, and data shown are from 5 mice per group. Bars represent mean \pm SEM. ${ }^{* *} P<0.01,2$-way ANOVA with post hoc Tukey's test.

chain linker strongly affected the ability of MT-SP1 to cleave the DVD. Partial cleavage of the LC linker was only seen after extension by at least 2 amino acids, and full cleavage required extension by 3 amino acids (Figure 3B and the uncut gel found in Supplemental Figure 2). In contrast, cleavage of the HC linker could be achieved by addition of only a single amino acid, and this was the case for all designs containing an extended $\mathrm{HC}$ linker. Based on these data, we selected a DVD containing a 7-amino acid HC linker and an 8-amino acid LC linker for further evaluation. We found that this DVD is able to bind to PSCA-overexpressing cell lines identically to the parental $10 \mathrm{E} 8 \mathrm{mAb}$ and that this binding was completely lost after the DVD was incubated with MT-SP1 (Figure 3C). Additionally, incubation of the DVD with MT-SP1 allowed for anti-CTLA4 binding to reach the same level as the original anti-CTLA4 Ab (24H2 clone) (Figure 3D). The activation of the PSCA/CTLA4 DVD by cleavage from MT-SP1 increased the CTLA4-neutralizing activity of the molecule by 25 -fold compared with the intact form (Figure 3E). Together, these data led us to select a candidate PSCA/CTLA4 DVD version of the engineered anti-CTLA4 Ab (anti-CTLA4 DVD) with the following structure: a mouse IgG2a backbone with ODs derived from 10E8, IDs derived from 24H2, a 7-amino acid HC linker, and an 8-amino acid LC linker.

Bispecific engineered CTLA4 blockade enhanced tumor-specific targeting and retention. To compare the biodistribution of anti-CTLA4 and anti-CTLA4 DVD in vivo, the Abs were conjugated to the chelator desferrioxamine $\mathrm{B}$ (DFO) via solvent-exposed $\varepsilon$ amino groups on lysine side chains. The DFO-conjugated Abs were coupled to ${ }^{89} \mathrm{Zr}$ using previously reported conditions (16). Evaluating the biodistribution of ${ }^{89} \mathrm{Zr}$-anti-CTLA4 in intact male C57BL6/j mice with s.c. TRAMP-C2 injection by PET/CT showed that the radiotracer accumulated in the tumor over 8 to 72 hours, while the radiotracer retention in the blood generally declined over 8 to 72 hours, as expected (Supplemental Figure 3). The tumor-to-muscle ratio was approximately 6:1 at 48 hours after injection. Moreover, the activity detected in the bone was low at all time points (e.g., $2.57 \% \pm$ $0.4 \% \mathrm{ID} / \mathrm{g}$ at 48 hours after injection), underscoring the stability of the radiotracer in vivo. Tumor uptake of ${ }^{89} \mathrm{Zr}$-anti-CTLA4 was also significantly reduced by heat denaturing the construct prior to i.v. injection, demonstrating that the accumulation of ${ }^{89} \mathrm{Zr}$-anti-CTLA4 in the TRAMP-C2 tumors is likely due to specific receptor binding (Supplemental Figure 4). Next, we compared the biodistribution of ${ }^{89} \mathrm{Zr}$-anti-CTLA4 and ${ }^{89} \mathrm{Zr}$-anti-CTLA4 DVD in mice bearing s.c. TRAMP-C2 tumors. At 48 hours after injection, the tumor uptake of ${ }^{89} \mathrm{Zr}$-anti-CTLA4 DVD was approximately 1.6 -fold higher than what was observed for ${ }^{89} \mathrm{Zr}$-anti-CTLA4 (Figure 3, F and G; $P<0.05$ ). Overall, our data indicate that localization of the engineered antiCTLA4 DVD within the tumor microenvironment is significantly higher when compared with that of the original anti-CTLA4 Ab.

Reduced toxicity in vivo with anti-CTLA4 DVD. In order to test the toxicity associated with the anti-CTLA4 DVD Ab, we performed the adoptive transfer of naive $\mathrm{CD} 4^{+} \mathrm{T}$ cells $\left(\mathrm{CD} 4^{+}\right.$ 
$\mathrm{CD} 5 \mathrm{RB}^{\mathrm{hi}} \mathrm{CD}^{-} 5^{-}$) into $\mathrm{Rag} 1^{-/-}$recipient mice and treated those mice with either parental anti-CTLA4 or anti-CTLA4 DVD Ab. As can be seen in patients with irAEs (17), we saw signs of lethargy and diarrhea, correlating with previously observed clinical symptoms, accompanied by body weight loss over time in mice that received anti-CTLA4 (Figure $4 \mathrm{~A}$ ). In contrast, mice that received anti-CTLA4 DVD did not exhibit signs of body weight loss over time, showing a significant improvement compared with the original anti-CTLA4 treatment (Figure 4A; $P<0.01$ ). The irAEs associated with ipilimumab treatment also presented with multiorgan pathogenesis. Inflammatory hepatitis has been reported in approximately $2 \%-5 \%$ of the patients who received CTLA 4 blockade (18, 19). In our anti-CTLA4-treated mice, we also observed multifocal lymphocytic infiltration in the parenchymal hepatic tissue and around the portal area, which was similar to what is seen in patients (20). Moreover, the lymphocyte infiltrations were accompanied by mild tissue destruction. In contrast, mice treated with anti-CTLA4 DVD had intact hepatic architecture, with only scarce amounts of lymphocytes present in the liver (Figure 4B; liver section). Pulmonary toxicity has also been reported in patients receiving ipilimumab treatments $(21,22)$. In our study, we also observed antiCTLA4-mediated lung toxicities associated with perivascular and peribronchial lymphocyte aggregates leading to bronchiolitis and pneumonitis. This pathological change, however, was reduced in mice receiving anti-CTLA4 DVD (Figure 4B; lung section). Endocrinopathies associated with CPI $(23,24)$ were also observed, with multifocal tissue destruction in the pancreas after anti-CTLA4 treatments in our murine model. This tissue destruction was ameliorated in mice treated with anti-CTLA4 DVD (Figure 4B; pancreas section). Additionally, anti-CTLA4 treatment induced marked diffuse lymphocytic infiltration, with tissue destruction in the GI tract, but the pathological changes were reduced in mice that received anti-CTLA4 DVD (Figure 4B; GI tract section). Overall, our data demonstrated that mice receiving anti-CTLA4 exhibited multiorgan toxicities similar to the irAEs observed in the clinic and that, by administering anti-CTLA4 DVD, such unwanted pathological changes can be avoided.

Maintenance of tissue Tregs with anti-CTLA4 DVD. The efficacy of anti-CTLA4 in murine tumor models requires activation of effector T cells and ADCC activity against Foxp $3^{+}$Tregs to mediate antitumor activity (25), yet preservation of Tregs in peripheral tissues can prevent development of irAEs (26). To investigate the Treg population, immunohistochemistry was performed. We found that there were higher percentages of Foxp ${ }^{+}$cells surrounding the glandular epithelium of the GI tract in the negative control and anti-CTLA4 DVD-treated groups compared with the anti-CTLA4-treated group, reflecting significantly degraded Tregs in the GI tract in this latter treatment group (Figure 5A). To further evaluate the ability of anti-CTLA4 DVD in limiting systemic depletion of Tregs, we utilized a T cell-mediated colitis model (27). In this model, the transfer of naive $C D 4^{+} C D 45 R^{\text {hi }}$ $\mathrm{T}$ cells from $\mathrm{BALB} / \mathrm{c}$ into SCID mice induces colitis that can be prevented by cotransfer of $\mathrm{CD} 4{ }^{+} \mathrm{CD} 45 \mathrm{RB}^{\text {lo }}$ cells due to the presence of Tregs from the latter population $(28,29)$. Endoscopic evaluation of the recipient mice at day 35 after adoptive transfer showed that the majority of animals receiving naive $\mathrm{T}$ cells alone $\left(C D 4{ }^{+} C D 45 \mathrm{RB}^{\mathrm{hi}}\right)$ developed severe colitis with an average murine endoscopic disease activity index (MEDAI) score of 9 (see Methods). In contrast, the cotransfer of $\mathrm{CD} 4{ }^{+} \mathrm{CD} 45 \mathrm{RB}^{\text {lo }}$ cells prevented colitis development (Figure 5, B and C). However, the protective effects of $\mathrm{CD}^{+} \mathrm{CD} 45 \mathrm{RB}^{\text {lo }}$ cells were completely abrogated with biweekly treatments of anti-CTLA4 for the first 3 weeks following cotransfer. This change was due to the depletion of CTLA4 ${ }^{+}$cells by ADCC, since treatment of an anti-CTLA $4 \mathrm{Ab}$ with a mutated Fc region (anti-CTLA4 DANA; 24H2 clone) that lacks Fc binding did not lead to disease (Figure $5 \mathrm{C}$ ). Yet when the cotransferred mice were treated with anti-CTLA4 DVD, $60 \%$ of the mice were protected from the development of colitis, while all of the mice in the anti-CTLA4 treatment group succumbed to colitis. Next, we examined the Treg population and found that the anti-CTLA4-treated group had significantly reduced tissue-resident Tregs compared with the isotype group (Figure $5 \mathrm{D} ; P<0.05$ ), while anti-CTLA4 DANA or anti-CTLA4 DVD treatments preserved Tregs. Taken together, these data demonstrate that shielding the CTLA4 ID in the tumor-conditional DVD is an effective approach for limiting systemic immune activation from CTLA4 blockade and avoiding ADCC-mediated Treg depletion in peripheral tissues.

Upregulation of ICOS on circulating T cells can be a clinically relevant biomarker of immune activation in patients treated with anti-CTLA4 (30). To investigate the ability of anti-CTLA4 DVD to limit systemic immune activation in vivo, we used flow cytometry to examine ICOS expression in mice treated with anti-CTLA4 or anti-CTLA4 DVD (Figure 5E). Administration of anti-CTLA4 Ab to WT mice resulted in a dose-dependent upregulation of ICOS on both splenic $\mathrm{CD}^{+}$and $\mathrm{CD}^{+}{ }^{+} \mathrm{T}$ cells (Figure $5, \mathrm{~F}$ and $\mathrm{G}$ ). At the highest dose, anti-CTLA4 Ab resulted in more than $40 \%$ of ICOS expression on $\mathrm{CD}^{+} \mathrm{T}$ cells as opposed to more than $12 \%$ in the anti-CTLA4 DVD-treated group. A similar trend was also observed in $\mathrm{CD}^{+} \mathrm{T}$ cell subsets. Overall, our data demonstrated that the inner domain conditional silencing in the DVD design is sufficient to prevent systemic $\mathrm{T}$ cell activation.

Anti-CTLA4 DVD preserved antitumor activity compared with CTLA4 blockade. Although prevention of systemic Treg depletion is important for preventing multiorgan toxicities, Treg depletion in tumors is essential for triggering antitumor immunity in murine models (10). Additionally, the therapeutic effect of anti-CTLA4 has also been demonstrated in the $\mathrm{CD} 4^{+}$effector $\mathrm{T}$ cell compartment within the tumor microenvironment (25). To further examine the details of the immunological changes in tumor-bearing mice treated with anti-CTLA4 and anti-CTLA4 DVD, we utilized the TRAMP-C2 tumor model expressing PSCA, which can be targeted by the antigen-binding site of the ODs of the Ab (Figure 6A). Mice were implanted with TRAMP-C2 tumors and treated with different CTLA4 blockades. Both anti-CTLA4 and anti-CTLA4 DVD significantly inhibited tumor growth compared with either PSCA alone or isotype control (Figure 6, B and C). Also, mice treated with anti-CTLA4 or anti-CTLA4 DVD exhibited prolonged survival in the TRAMP-C2 tumor challenge experiment (Figure 6D). In the dose-response experiment, both anti-CTLA4 and anti-CTLA4 DVD demonstrated similar tumor inhibitory effects in TRAMP-C2bearing mice (Supplemental Figure 5, A and B). To further investigate the immune landscape within the tumor microenvironment, tumors were harvested from checkpoint inhibitor-treated mice and analyzed by flow cytometry (Figure 7A). We found that anti- 
A

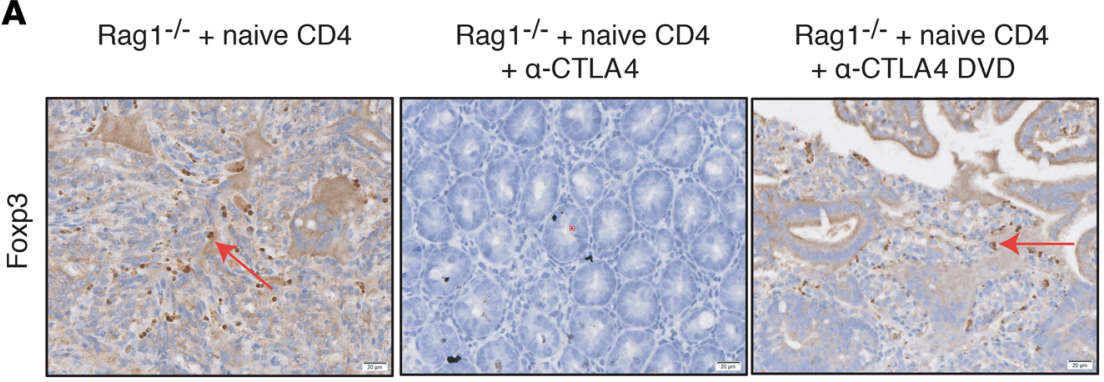

C

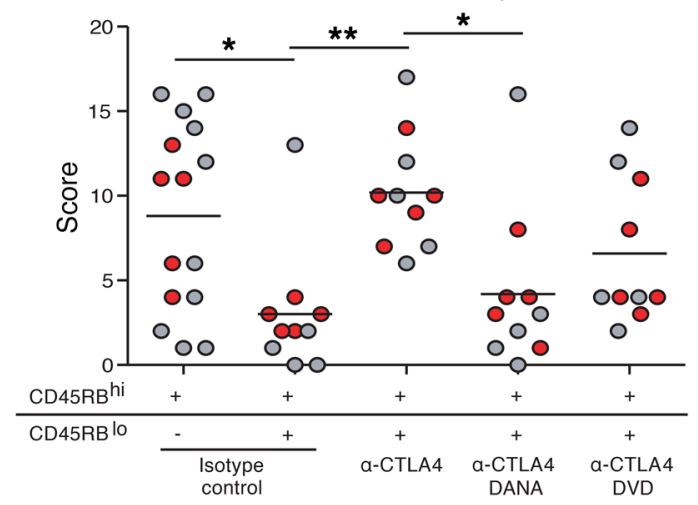

B

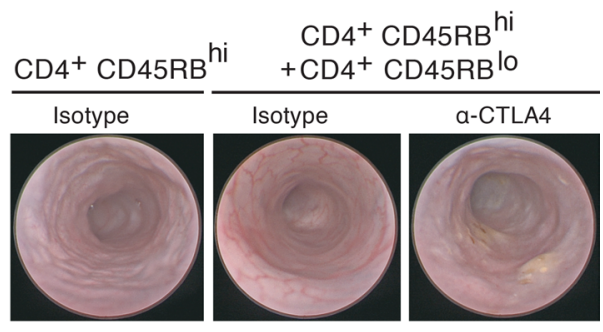

a-CTLA4 DANA a-CTLA4 DVD

D

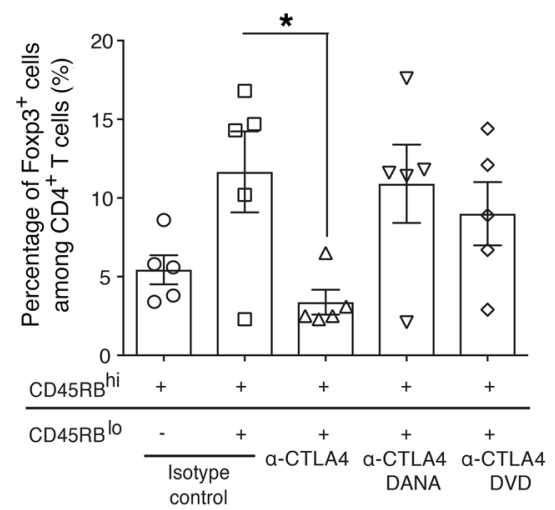

E

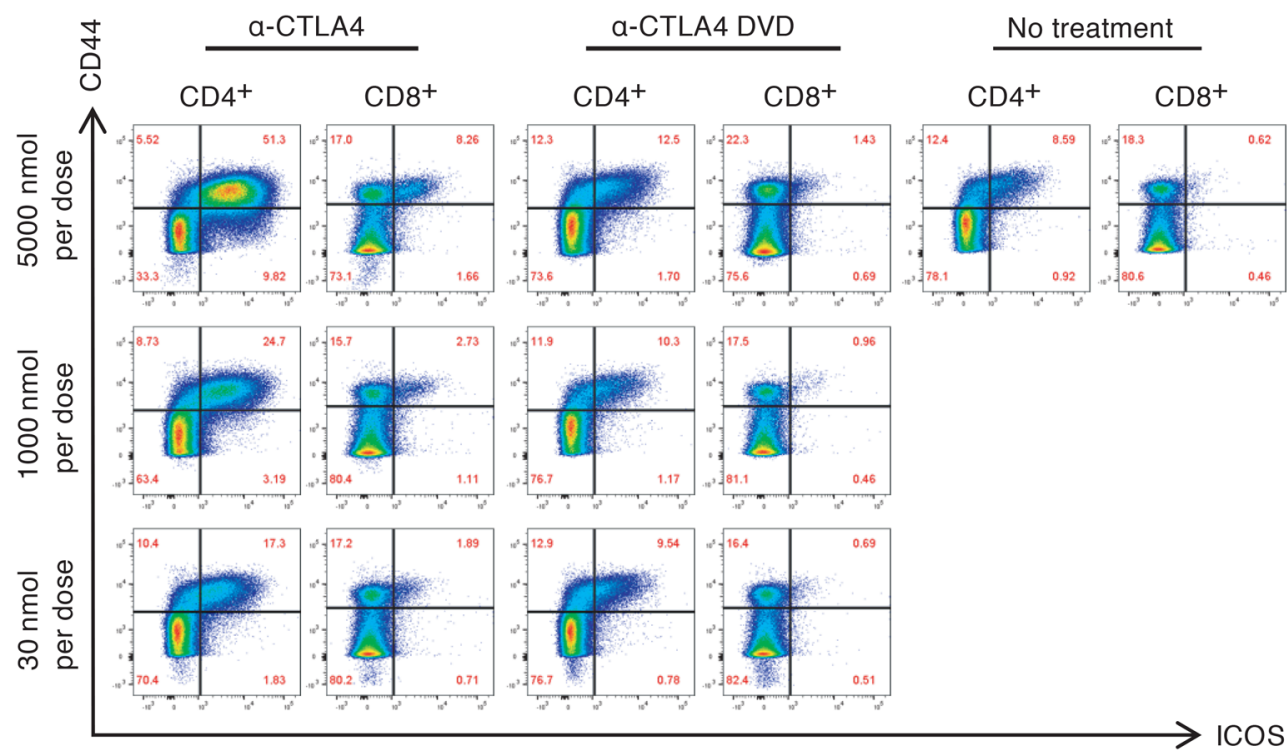

$\mathbf{F}$

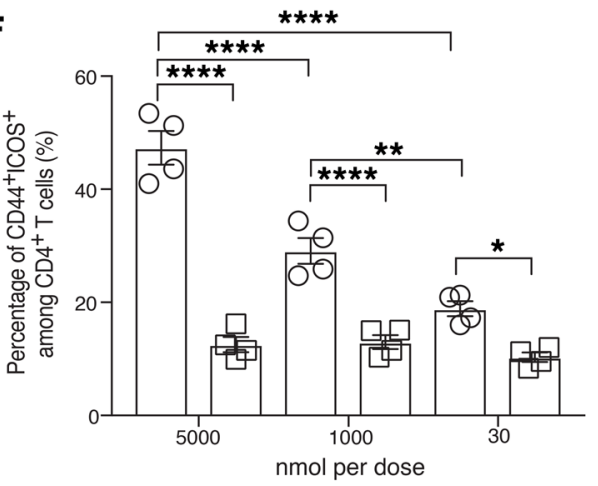

G

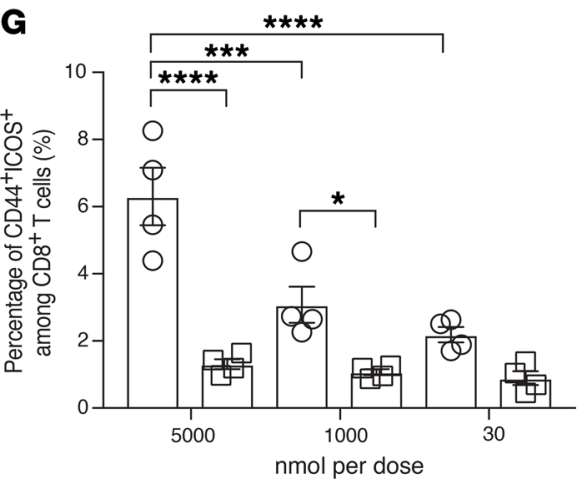

O a-CTLA4

$\square$ a-CTLA4 DVD 
Figure 5. Colitis induction following treatment with anti-CTLA4, but not with anti-CTLA4 DVD. (A) Foxp3 IHC staining was performed on GI tract tissue sections from mice receiving different Abs. Arrows indicate Foxp3 ${ }^{+}$ cells. Experiments were conducted twice with 6 mice per group, and the representative figure from each treatment arm was shown. (B-D) Recipient C.B17 SCID mice were i.p. injected with $5 \times 10^{5}$ purified CD4+CD45RB hi cells with or without $2.5 \times 10^{5} \mathrm{CD}^{+}{ }^{+} \mathrm{CD} 45 \mathrm{RB}^{\text {lo }}$ cells, and administered the indicated treatments at $200 \mu \mathrm{g}$ per dose twice per week for 3 weeks. At day 35 , all mice were evaluated by endoscopy and scored for colitis. Data were collected from 1 experiment with 10-15 mice per treatment arm. (B) Representative endoscopy images of a single mouse from each group. (C) Graphic representation of endoscopy scores at day 35 . Red dots indicate animals from which colons were further analyzed for percentage of $\mathrm{Foxp}^{+}$cells among $\mathrm{CD}^{+}$cells in D. (E-C) Healthy C57BL/6j mice were injected with either anti-CTLA4 or anti-CTLA4 DVD. (E) Flow-gating strategy of spleen samples. (F) Percentage of $\mathrm{CD}_{4} 4^{+} \mathrm{ICOS}{ }^{+}$in $\mathrm{CD}^{+}{ }^{+} \mathrm{T}$ cell subsets. (C) Percentage of $\mathrm{CD} 44^{+} \mathrm{ICOS}^{+}$in $\mathrm{CD} 8^{+} \mathrm{T}$ cell subsets. For parts $\mathbf{E}-\mathbf{G}$, experiments were conducted twice, and the representative data from 1 experiment was from 4 mice per group. Bars represent mean or mean \pm SEM. ${ }^{*} P<0.05 ;{ }^{* *} P<0.01 ;{ }^{* *} P<0.001 ;{ }^{* * *} P<0.0001$, 1 -way ANOVA with post hoc Tukey's test.

CTLA4 increased the density of tumor-infiltrating $\mathrm{CD}^{+}{ }^{+} \mathrm{T}$ cells compared with anti-PSCA or isotype control treatments (Figure 7B). Both anti-CTLA4 and anti-CTLA4 DVD successfully increased ICOS expression in effector $\mathrm{CD} 4^{+} \mathrm{T}$ cells (Figure $7 \mathrm{C}$ ) while depleting tumor-infiltrating Tregs compared with isotype control or antiPSCA alone (Figure 7D). Treatment with both classes of CTLA4 blockades also induced higher amounts of tumor-infiltrating CD8 ${ }^{+}$ T cell subsets than the control group (Figure 7E; $P<0.01$ and $P<$ 0.05 , respectively), and those $C D 8^{+} \mathrm{T}$ cells had higher expression of CD25 (Figure 7F; $P<0.01$ and $P<0.05$, respectively) and Ki-67 (Figure 7G; $P<0.01$ ) compared with isotype-treated mice, indicating their highly activated status and proliferative capabilities. Spas-1 represents an immunodominant epitope in the TRAMP-C2 tumor model (14). Here, we found that mice treated with anti-CTLA4 DVD had significantly increased frequency of tumor-infiltrating $C D 8^{+} \mathrm{T}$ cells that recognize Spas-1 compared with either the PSCA-alone group $(P<0.01)$ or the isotype-treated group (Figure $7 \mathrm{H} ; P<0.05)$.

The CTLA4 costimulation pathway is also involved in early $\mathrm{T}$ cell activation in peripheral lymphoid organs (31). To further investigate the systemic antitumor immune responses of different CTLA4 blockades, spleen and draining lymph nodes were harvested after treatments and analyzed by flow cytometry (Supplemental Figure 6A). In draining lymph nodes, anti-CTLA4 significantly induced higher amounts of total CD $4^{+} \mathrm{T}$ cells and ICOS expression compared with anti-CTLA4 DVD, anti-PSCA, or isotype control (Supplemental Figure 6, B-D). Similarly, $\mathrm{CD}^{+}$and Spas-1-reactive $\mathrm{CD}^{+} \mathrm{T}$ cells were also increased in the anti-CTLA4-treated group, but not in the anti-CTLA4 DVD, anti-PSCA, or isotype control groups (Supplemental Figure 6, E and F). Similar trends were also observed in the spleen. While anti-CTLA4 induced higher amounts of $\mathrm{CD}^{+}$and $\mathrm{CD} 8^{+} \mathrm{T}$ cells systemically, the numbers were significantly reduced in the anti-CTLA4 DVD group, particularly with the $\mathrm{CD}^{+} \mathrm{T}$ subset (Supplemental Figure 6, G-K). Overall, these data supported the notion that the CTLA4 neutralizing activity of the PSCA/CTLA4 DVD is conditionally inhibited to prevent systemic immune activation in lymphoid organs, while maintaining antitumor activity by localized depletion of Tregs and increased $\mathrm{CD}^{+} \mathrm{T}$ cells within the tumor (Figure 8).

\section{Discussion}

irAEs have emerged as a major barrier that limits the administration of CPI, including the number of doses that a patient can receive. In a recent phase III trial, grade $3 / 4$ irAEs occurred in $28 \%$ of the patients who received ipilimumab treatment (anti-CTLA4). When anti-CTLA4 is combined with anti-PD-1 Abs, the frequency of irAEs can rise to around $60 \%$ (5). As a result, there is an increasing need to monitor and manage irAEs found in the clinic. In this study, we created an "antigen-specific" CTLA4 blockade that is locally released in the tumor microenvironment, thus minimizing systemic exposure. We found that limiting the exposures of CTLA4 blockade can preferentially deplete tumor Tregs and enhance infiltration of antigen-specific $\mathrm{T}$ cells while preventing systemic activation of $\mathrm{CD}^{+}$or $\mathrm{CD}^{+} \mathrm{T}$ cell subsets. Compared with the original CTLA4 blockade, mice treated with this new Ab did not exhibit symptoms associated with treatment toxicities in organs, including the liver, GI tract, lung, and pancreas. Additionally, this new class of tumor-conditional anti-CTLA4 provided antitumor immunities similar to those found with the original CTLA4 blockade. Hence, the format of antigen-binding anti-CTLA4 DVD provides a potential clinical application with strong antitumor efficacy and reduced risks of irAEs.

Several mechanisms have been linked to CTLA4-mediated toxicities (32). During naive $\mathrm{T}$ cell activation, the expression of CTLA4 protein on the cell surface is upregulated and directly competes with the CD28 protein in binding with the CD80 and CD86 molecules (31). The inhibitory signals from CTLA4 can also prevent the activation of self-reactive $\mathrm{T}$ cells by attenuating $\mathrm{T}$ cell signaling (33). Additionally, systemic exposure of anti-CTLA4 in patients leads to an expansion of $\mathrm{T}$ cell receptor clonality that is associated with clinical symptoms of irAEs (11). Therefore, to prevent multiorgan toxicities, limiting the systemic exposure of CTLA4 blockade may be beneficial. Recent studies have also shown that localized administration of CTLA4 blockade in tumors was sufficient in preserving antitumor effects, illustrating that limiting systemic exposure does not affect the efficacy of CTLA4 blockade $(13,34)$. Based on these studies, we developed an "antigen-specific" CTLA4 blockade that can be systemically delivered without triggering toxicities, making it a potential clinical platform for targeting different arrays of tumor antigens.

Foxp ${ }^{+}$Tregs are one of the major cell types required for maintaining tolerance and are known for high expression of surface CTLA4 (35). In preclinical models, administration of anti-CTLA4 successfully controlled tumor growth (36) and depleted Tregs via Fc-dependent ADCC activity (10). However, systemic abrogation of Tregs mediated by the CTLA4 blockade can also induce hyperproliferation of lymphocytes that manifest into multiorgan autoimmune disease-like symptoms, including pneumonitis, insulitis, and gastritis (37). Therefore, limiting CTLA4 blockade to the tumor microenvironment could be essential in exerting potent antitumor effects while maintaining peripheral tolerance. Our approach relies on 2 components to localize anti-CTLA4 to the tumor site. First, the DVD OD binds to a tumor-associated antigen, leading to the preferential accumulation of the $\mathrm{Ab}$ at the tumor site. Second, we utilized the tumor-associated expression of the protease MT-SP1. Upon engagement with the enzyme, the outer shield was cleaved, releasing the inner domain of the 
A

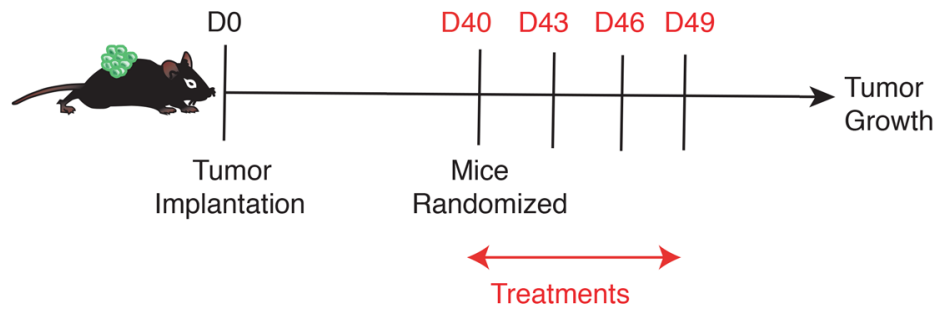

B

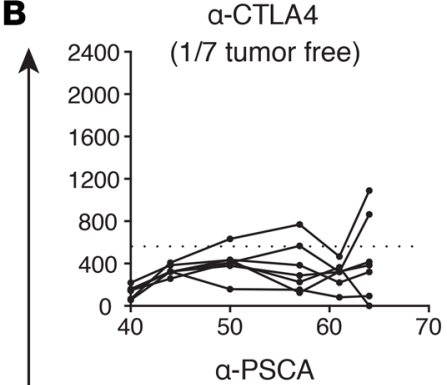

a-CTLA4

ต

\begin{abstract}
a-PSCA
\end{abstract} (0/7 tumor free)

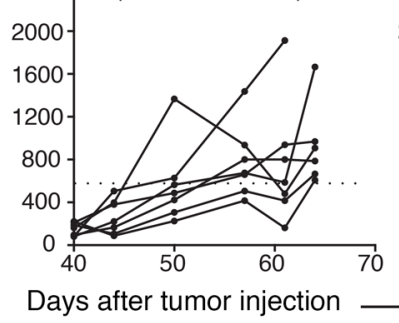

c

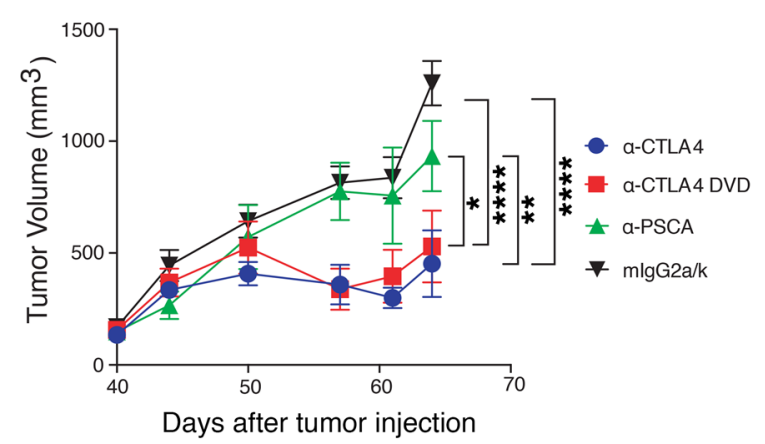

D

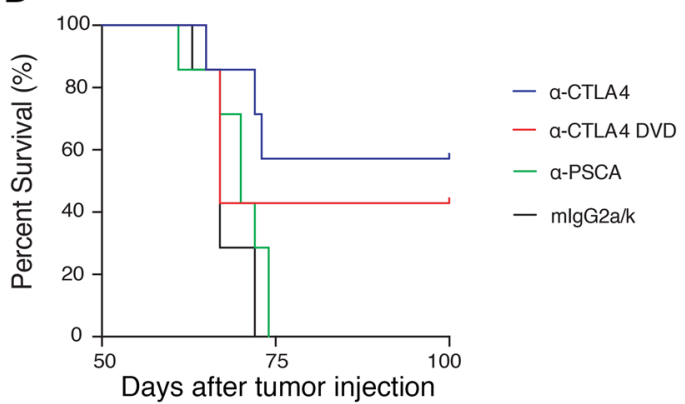

Figure 6. Anti-CTLA4 DVD preserves antitumor activity. (A) C57BL/6j male mice were implanted with TRAMP-C2 tumors at day 0 (D0), and tumors were allowed to grow for 40 days. Mice were randomized into different treatment groups before Ab injection and then received different treatments at days 40 , 43, 46, and 49. (B and C) Tumor growth from different treatment groups over time. (D) Survival curve of different treatment groups. Data were conducted with 2 independent experiments. Each treatment arm was collected from 7 mice per group. Bars represent mean $\pm \mathrm{SEM}$. ${ }^{*} P<0.05$; ${ }^{* *} P<0.01 ;{ }^{* * * *} P<$ $0.0001,2$-way ANOVA with post hoc Tukey's test.

CTLA4-binding site. We found that this approach of CTLA4 $\mathrm{Ab}$ design is able to preferentially deplete only tumor-infiltrating Tregs while preserving the Tregs in peripheral tissues, thus avoiding irAEs. Importantly, splenic T cell expansion/activation associated with anti-CTLA4 in our tumor model is spared with anti-CTLA4 DVD treatment, suggesting that escape of tumoractivated Abs is minimal in this model. While we observed a potent antitumor activity from the anti-CTLA4 DVD treatment, the therapeutic efficacy may also be contributed by the outer PSCA-targeting domain, as the previous report has demonstrated the tumor-inhibitory effects of anti-PSCA (38).

During $\mathrm{Ab}$ development, the selection of Ig configuration is crucial in determining the efficacy of the Ab through its Fc receptor activity $(39,40)$. Ipilimumab is an IgG1-based CTLA4 blockade and has been shown to mediate Treg depletion ex vivo via Fc $\gamma$ RIIIA (41). However, Treg depletion in cancer patients with ipilimumab has rarely been observed, and the clinical activities associated with ipilimumab are more prone to alter conventional CD4 ${ }^{+}$T cells $(11,42,43)$. This is in contrast with murine studies, in which antitumor effects of CTLA4 blockade are mainly mediated by reducing Tregs within tumors (10). Different clones of CTLA4 blockade in mice have distinct capabilities in Treg depletion, as shown by the $9 \mathrm{H} 10$ and $24 \mathrm{H} 2$ clones, which are better at depleting Tregs through ADCC compared with the UC-10 clone of antiCTLA4 Ab (44). In our study, the antitumor activity of the CTLA4 DVD blockade was built upon the mouse IgG2a isotype (24H2 clone), in which the therapeutic activity partially relied on the Fc $\gamma$ receptor for intratumoral Treg depletion via ADCC. However, the lack of a human equivalent ADCC-competent isotype may limit such an approach in the clinic (43). By using humanized Fc receptor transgenic mice, one can potentially bridge the gap between preclinical Ab development and the clinical CTLA4 blockade by accounting for the Fc-binding difference between species in future studies (45). As the therapeutic response of ipilimumab is associated with the high affinity of Fc $\gamma \mathrm{R}$ polymorphism (46) and ADCC requires natural killer cells and myeloid cells (47), enhancement of Fc-binding capability in treating "inflamed" tumors can potentially be more suitable for such engineered tumor-targeting CTLA4 blockades. Thus, our current approach provides a useful rationale for dissociating immune toxicities from antitumor activities, but caution must be taken into consideration when choosing an ADCC-competent isotype and treating inflamed tumors in the clinic. Finally, the potential immunogenicity of the engineered DVD will also need to be examined. 
A

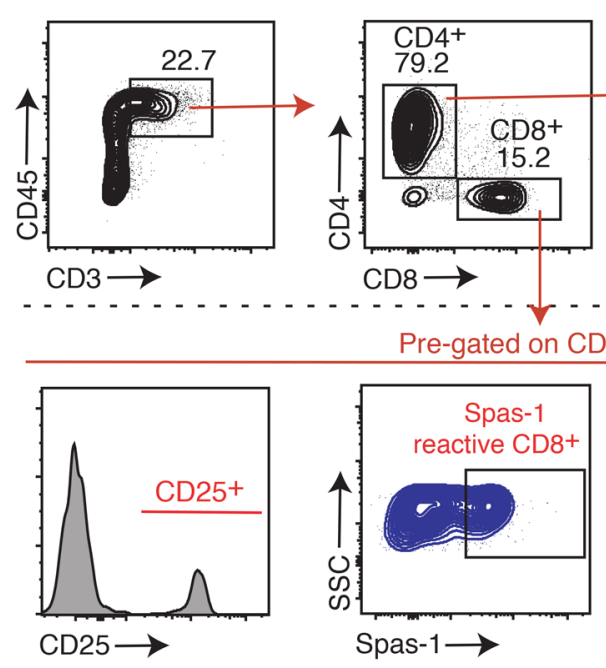

C

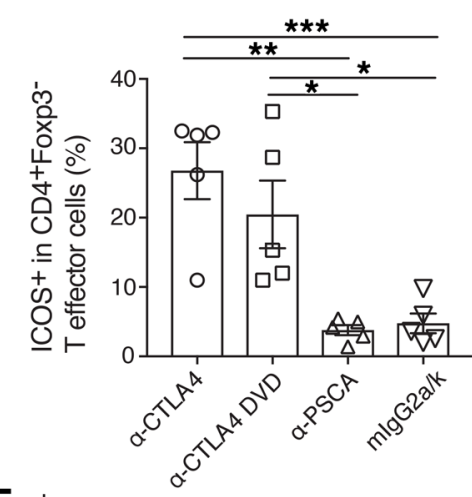

$\mathbf{F}+$

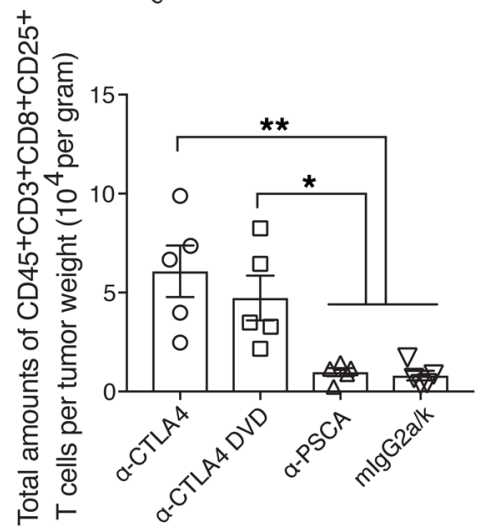

D

G
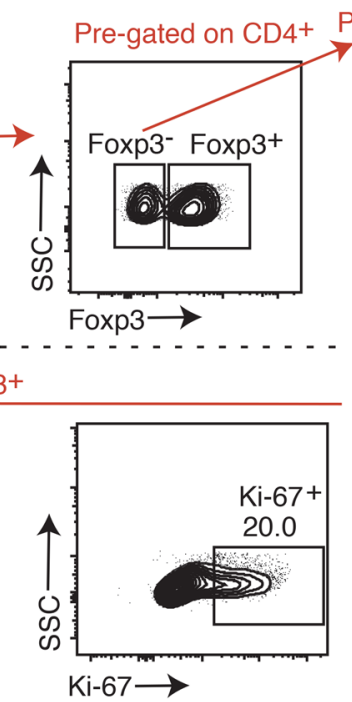

Pre-gated on CD4+Foxp3-
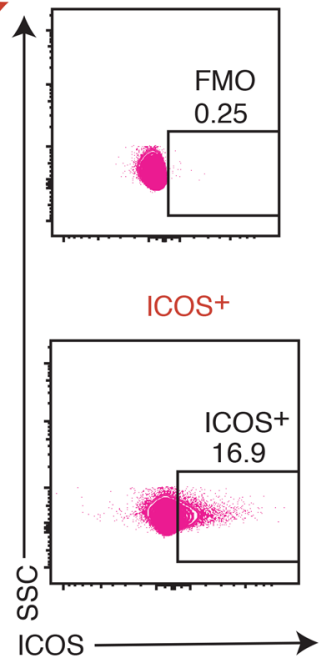

B

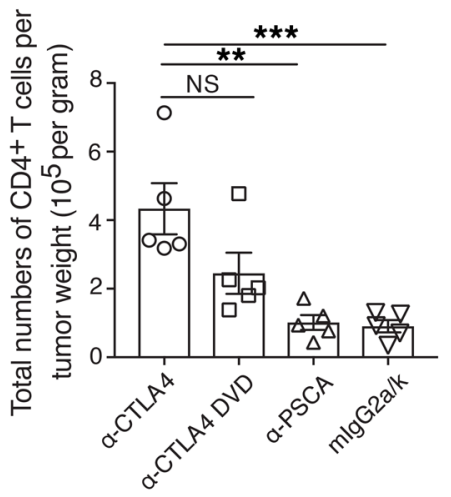

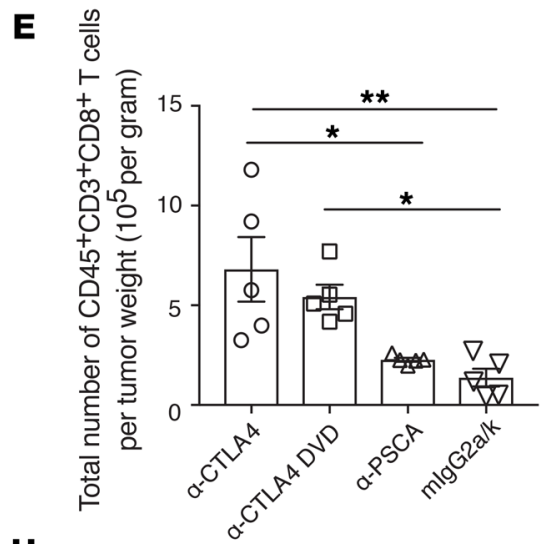

H
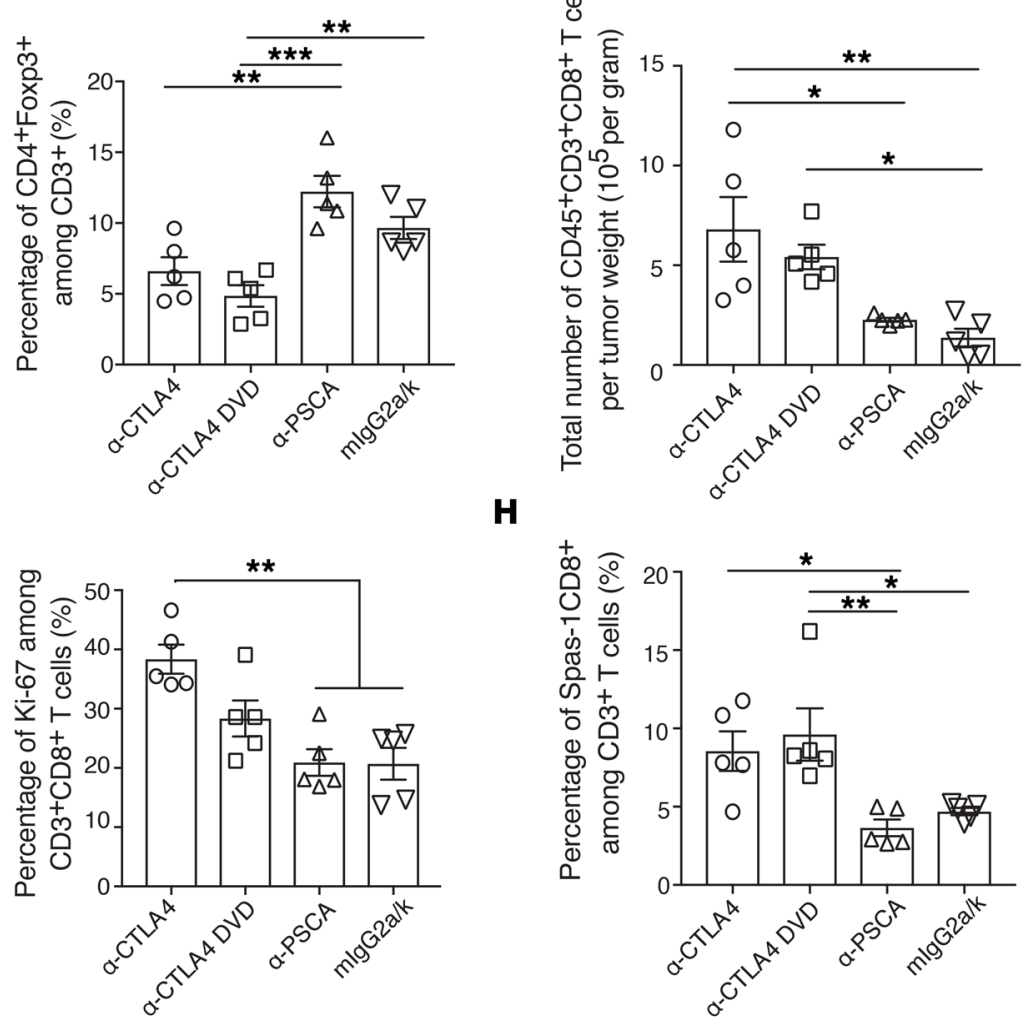

Figure 7. Immune landscape within tumors after different CTLA4 blockades. C57BL/6j male mice were implanted with TRAMP-C2 tumors at day 0, and tumors were allowed to grow for 40 days. Mice were randomized into different treatment groups and received different treatments at days $40,43,46$, and 49. Tissues were harvested at day 52 and analyzed by flow cytometry. (A) Tumors were harvested from each treatment group and analyzed for different immune subsets by flow cytometry. (B) Total numbers of $C D 4^{+} T$ cells per tumor weight. $C D 4^{+} T$ cells were pregated on live $C D 45^{+} C D 3^{+} C D 8^{-} T$ cells. (C) Percentage of ICOS expression among CD4+Foxp3- T cells. (D) Percentage of CD4+Foxp3 ${ }^{+}$among $\mathrm{CD}^{+}$cells. (E) Total number of infiltrating CD8 ${ }^{+} \mathrm{T}$ cells per tumor weight. (F) Total numbers of CD8 ${ }^{+} C D 25^{+} T$ cells per tumor weight. (G) Percentage of Ki-67 among CD3+CD8 ${ }^{+} T$ cells. (H) Percentage of Spas-1-reactive $\mathrm{CD}^{+} \mathrm{T}$ cells among $\mathrm{CD}^{+}$cells. Two independent experiments were conducted. Data were shown as 5 mice per group from 1 representative experiment. Bars represent mean \pm SEM. ${ }^{*} P<0.05 ;{ }^{* *} P<0.01$; ${ }^{* * *} P<0.001$, 1-way ANOVA with post hoc Tukey's test.

In summary, we reported a class of anti-CTLA4 $\mathrm{Ab}$ whose pharmacology acts in a "tumor targeted" way by enhancing antitumor activities while minimizing potential toxicities. With the increasing concerns of high-grade toxicity associated with anti-CTLA4 usage in the clinic, this engineered antiCTLA4 DVD Ab provides powerful insights for designing the next generation of CPI to achieve more promising clinical outcomes for future patients. 
(1) Silenced inner domain minimizes toxicity when the antibody circulates systemically
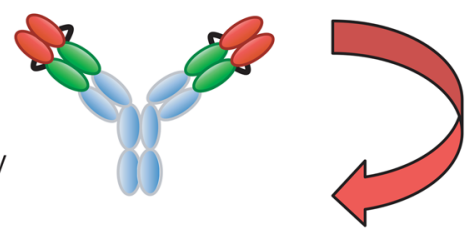

(2) Systemic circulation of antibody can target cancer

cells by outer antigen-targeting domain
Tumor microenvironment

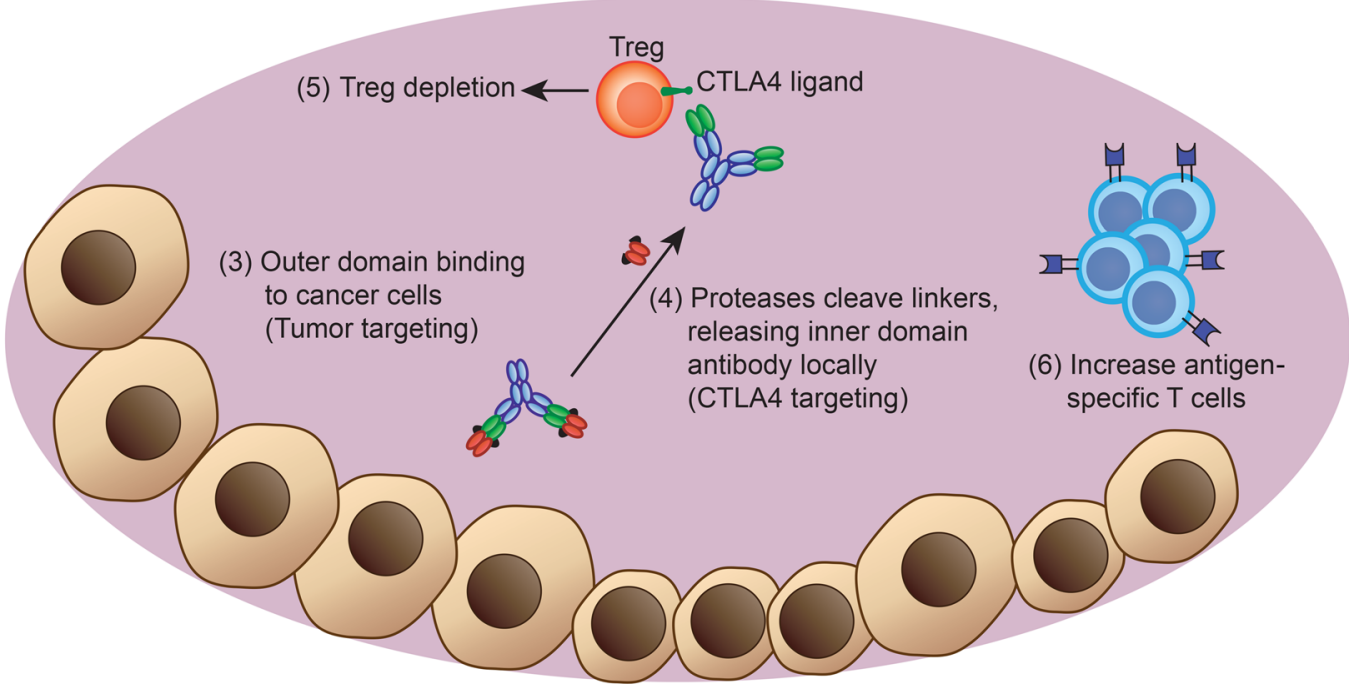

Figure 8. Diagram of engineered anti-CTLA4 DVD blockade in vivo. The engineered $A b$ consists of an outer domain and an inner domain. During systemic circulation, the CTLA4-binding site is shielded by the outer PSCA-binding domain. The anti-PSCA can then guide the $A b$ and accumulate into the tumor microenvironment, where the protease can cleave the linker and expose the inner CTLA4-binding site. The anti-CTLA4 DVD can then deplete Treg cells and activate effector T cells within tumors to achieve antitumor activities.

\section{Methods}

Generation of anti-CTLA4 Abs. The mouse anti-CTLA4 Ab (clone 24H2) and anti-CTLA4 DVD (clone 24H2) and anti-PSCA Ab (clone 10E8) were generated by AbbVie. The cDNA of variable domain from HC and LC of the 2 hybridoma clones was amplified by RACE PCR using Ig constant region-specific primers. The variable domains were converted and expressed as rat anti-mouse IgG2a chimeric Abs and used for subsequent studies as controls for the DVD Ig derived from them. DVD Ig constructs were generated as described previously $(48,49)$. Briefly, the $\mathrm{VH}$ and VL sequences of the PSCA Ab as outer domains and the VH and VL sequences of the CTLA4 Ab as inner domains were linked with a cleavable linker (LSGRSDNH or optimized linker) and expressed with mouse IgG2a heavy chain and $\kappa$ light chain domains. FACS was used to characterize the DVD Ig binding to PSCA or CTLA4 overexpressing HEK293.

Murine toxicity model by CTLA4 blockade. Eight- to ten-week-old male WT C57BL/6j and Rag 1/- mice (on C57BL/6j background) were purchased from Jackson Laboratory for the following mouse experiments. Splenocytes from WT mice were harvested and processed into single-cell suspension. Purified $\mathrm{CD}^{+} \mathrm{T}$ cells were then isolated by magnetic beads according to the manufacturer's protocol (Miltenyi Biotec, catalog 130-104-454). The CD $4^{+} \mathrm{T}$ cells were subsequently sorted on a CD25-CD45RB hi population by FACSAria, as indicated in the manuscript. After sorting, cells were checked for purity and viability. Each recipient mouse received $5 \times$ $10^{5}$ purified $\mathrm{CD} 4{ }^{+} \mathrm{CD} 25^{-} \mathrm{CD} 45 \mathrm{RB}^{\text {hi }}$ cells i.p. Anti-CTLA4 (24H2 clone), anti-CTLA4 DVD, anti-PSCA, and isotype control treatments were given i.p. from day 0 and repeated every 3 days at a dose of $200 \mu \mathrm{g}$ per mouse. Starting from day 12 , Abs were given at a maintenance dose of $100 \mu \mathrm{g}$ per mouse every 5 days. In the meantime, mice were monitored for body weight loss and clinical symptoms (diarrhea or lethargy) twice a week.

To evaluate $\mathrm{T}$ cell responses after CPI, 6- to 10-week-old male C57BL/6 mice were dosed once every 3 days for a period of 9 days by i.p. injection of Abs in PBS. Two days following the last dose, treated mice were sacrificed and spleens were harvested for analysis by FACS. For $\mathrm{T}$ cell transfer colitis, untouched $\mathrm{CD} 4^{+} \mathrm{T}$ cells were purified from the spleens of BALB/c mice by negative immunomagnetic selection (Stem Cell Technologies) and further fractionated into $\mathrm{CD} 4{ }^{+} \mathrm{CD} 45 \mathrm{RB}^{\mathrm{hi}}$ and $\mathrm{CD}^{+}{ }^{+} \mathrm{CD} 45 \mathrm{RB}^{\mathrm{lo}}$ subsets by FACS. Colitis was induced by i.p. injection of 5 $\times 10^{5} \mathrm{CD} 4^{+} \mathrm{CD} 45 \mathrm{RB}^{\text {hi }}$ cells into C.B17 SCID mice. Some animals received a 2:1 mixture of $\mathrm{CD} 4^{+} \mathrm{CD} 45 \mathrm{RB}^{\text {hi }}$ and $\mathrm{CD} 4^{+} \mathrm{CD} 45 \mathrm{RB}^{\text {lo }}$ cells. Recipient mice received i.p. injections of isotype $\mathrm{Ab}$, anti-CTLA4, anti-CTLA4 DANA, or anti-CTLA4 DVD twice a week for 3 weeks after adoptive transfer. All animals were evaluated by endoscopy at days 21 and 35 and scored for colitis using the MEDAI. The scoring system was based on changes in the vascular patterns, granularity of the mucosal surface, and the presence of exudates. Images of the colon were captured at 3, 2 and $1 \mathrm{~cm}$ from the anal verge. Each image was scored based on 3 parameters: Exudate $(0$ $=$ normal, $1=<50 \%$ area covered, $2=>50 \%$ of area covered), Vascular Changes $(0=$ normal, $1=$ vessels disconnected $/$ small vessels not visible, 2 = large vessels not visible/ starburst pattern, 3 = surface bleeding) and Mucosal Granularity $(0$ = normal, 1 = slight cobblestone appearance, 2 = pronounced and extensive cobblestone appearance, $3=$ mucosal protrusion, reduced lumen size). All scores were added together to obtain the Sum Score (maximum score $=24$ ). On day 36, all animals were sacrificed, and tissues were collected from 5 animals per group for analysis by FACS.

In vitro assessment of tumor matriptase activity and selection of anti-CTLA4 DVD Abs. Immunohistochemistry was performed for protease expression on TRAMP-C2 tumor sections. Fluorescent peptide probes containing the conditional DVD cleavable linker sequence were incubated with recombinant MT-SP1 at room temperature overnight. The fluorescent probes were tested in either TRAMP-C2 tumor lysate or tumor sections. To test the cleavage, SDS-PAGE was performed before or after coincubation of DVD Igs with MT-SP1 at a 1:10 dilution (w/w) overnight at room temperature. A DVD Ig made with linkers lacking the MT-SP1 cleavage site (LSGSDN/SGSDN) was used as control. To test the PSCA or 
CTLA4 binding, DVD Igs were incubated with MT-SP1 and added to an HEK293 cell line that overexpressed either PSCA or CTLA4. The specific binding was detected by FACS. To further detect blocking activity, intact or cleaved DVD Ig were incubated with HEK293 cells overexpressing CTLA4 in the presence of B7.2.Fc that was conjugated with Alexa Fluor 488 , and specific binding of B7.2.Fc to the cells was detected by FACS.

In vivo evaluation of antitumor activity by different CTLA4 blockades. Eight- to ten-week-old control male C57BL/6j mice were purchased from Jackson Laboratory and used in these experiments. The TRAMP-C2 cancer cells were injected s.c. on the right flank at a dosage of $1 \times 10^{6}$ cells per mouse on day 0 . The tumors were allowed to grow for $30-45$ days prior to treatment, and then mice with tumor volumes within $50-200 \mathrm{~mm}^{3}$ were randomized into different groups before starting treatment. The average tumor sizes among different treatment groups were checked and ensured to be similar before treatment. Mice were then treated with different anti-CTLA4 Abs (24H2 clone), anti-PSCA, or isotype control every 3 days for a total of 3 doses after randomization. Tumors were measured twice a week. Tumor volume was defined as follows: tumor volume $\left(\mathrm{mm}^{3}\right)=\mathrm{L}$ (length) $\times \mathrm{W}$ (width) $\times \mathrm{W} / 2$. More specifically, the longer diameter was defined as length, and the shorter diameter was defined as width. All mice were maintained at the UCSF vivarium.

Tissue preparation for flow cytometry. Spleens were surgically removed with sterilized surgical equipment and crushed with the blunt end of a 10 $\mathrm{ml}$ syringe on petri dishes containing $5 \mathrm{ml}$ of PBS. The resulting pieces of spleen were then crushed through a $70 \mu \mathrm{m}$ filter into a $50 \mathrm{ml}$ conical tube with more PBS. The spleen mixture was centrifuged at $450 \mathrm{~g}$ for 5 minutes at $4^{\circ} \mathrm{C}$ and then resuspended in $5 \mathrm{ml}$ red blood cell lysis solution (Santa Cruz Biotechnology Inc.; catalog sc-296258) on ice for 5 minutes. The reaction was stopped with the addition of $30 \mathrm{ml}$ PBS. After washing, cells were reconstituted for counting by Vi-CELL (Beckman Coulter). Draining lymph nodes were extracted with sterilized surgical equipment and crushed between the frosted surfaces of super-frosted microscope slides into wells containing PBS. Cell mixtures were then filtered through a 70 $\mu \mathrm{m}$ filter into $15 \mathrm{ml}$ conical tubes. Cells were then washed and counted. Tumors were removed from mice with sterile surgical instruments, followed by sectioning for paraformaldehyde fixation or flow cytometry analysis. Tumor tissues for flow analysis were kept moist with $1 \mathrm{ml}$ collagenase IV digest media (DMEM $+10 \% \mathrm{FCS}+1 \%$ penicillin/streptomycin + collagenase IV + DNase) and minced with scalpel blades. Tumor cell mixtures were then transferred into $15 \mathrm{ml}$ conical tubes and filled with an additional $9 \mathrm{ml}$ of collagenase digest media. Tumor samples were subsequently placed on a $37^{\circ} \mathrm{C}$ shaker for an hour of digestion. Samples were then filtered through a $100 \mu \mathrm{m}$ filter into a $50 \mathrm{ml}$ conical tube and washed with PBS before centrifugation. Finally, tumor cell pellets were resuspended and counted before subsequent $\mathrm{Ab}$ staining for flow cytometry.

Abs and flow staining. Single-cell suspensions (1 million cells/well) were first incubated with Fc Block (BD Biosciences - Pharmingen) for 10 minutes, then coincubated with Abs for 20 minutes at $4^{\circ} \mathrm{C}$, followed by washing with staining buffer (PBS $+1 \%$ FBS). Foxp3 and intracellular staining were performed using an eBioscience Intracellular Kit (catalog 00-5523-00) according to the manufacturer's protocol. Flow cytometry was performed on X-50 (BD) and data analyzed by FlowJo software (TreeStar). A detailed list of flow Abs can be found in Supplemental Table 1.

Histology and immunohistochemistry. Tissues harvested from mice were placed in $4 \%$ formalin, followed by $70 \%$ alcohol before embedding. Tissues were then embedded in paraffin, sectioned, and stained with H\&E. Tissue sections were evaluated by a board-certified pathologist.
Images were visualized using an Olympus Vanox AHBS3 microscope with an Olympus SPlan Apo ×20/0.70 NA objective (Olympus). A diagnostic instrument spot RT color digital camera utilizing Spot software, version 4.0.2, was used to acquire the images (Diagnostic Instruments). Immunohistochemistry was performed as described below. Tumor tissues were fixed in $4 \%$ paraformaldehyde, processed, embedded in paraffin, then cut into $5 \mu \mathrm{m}$ thick sections. For immunohistochemistry, paraffin sections were blocked with $3 \%$ hydrogen peroxide solution (MilliporeSigma, catalog H1009), vector streptavidin/biotin (Vector Laboratories, catalog SP-2002), and CAS-Block protein block (Thermo Fisher, catalog 008120), then stained with Foxp3 Ab (eBioscience, catalog 14-5773-82).

Radiolabeling of Abs. Anti-CTLA4 and anti-CTLA4 DVD (2 mg) was diluted in $1 \mathrm{ml}$ of PBS, and the $\mathrm{pH}$ was adjusted to 9.0 with $0.1 \mathrm{M}$ sodium bicarbonate buffer. In addition, $10 \mu \mathrm{l}$ of p-Isothiocyanatobenzyldesferrioxamine (Df-Bz-NCS) was added to the Ab solution. After 40 minutes at $37^{\circ} \mathrm{C}$, the reaction mixture was purified with a PD-10 column using a gentisic acid mobile phase $(5 \mathrm{mg} / \mathrm{ml}$ of gentisic acid in $0.25 \mathrm{M}$ sodium acetate, $\mathrm{pH}$ 5.4-5.6). The $\mathrm{pH}$ of the eluted DFO Ab solution was adjusted to 7.0 and stored at $-20^{\circ} \mathrm{C}$ until time of use.

A solution of ${ }^{89} \mathrm{Zr}$-oxalic acid ( $2 \mathrm{mCi}$ in $\left.3 \mu \mathrm{l}\right)$ was diluted with $190 \mu \mathrm{l}$ of $1 \mathrm{M}$ oxalic acid (Aqueous), and the $\mathrm{pH}$ of the solution was adjusted to pH 7.1-7.3 with $2 \mathrm{M} \mathrm{Na} 2 \mathrm{CO} 3(100 \mu \mathrm{l})$. After 3 minutes, $0.30 \mathrm{ml}$ of $0.5 \mathrm{M}$ HEPES, $0.5 \mathrm{mg}$ of DFO Ab ( $\mathrm{pH}=7)$, and $0.7 \mathrm{ml}$ of $0.5 \mathrm{M}$ HEPES were added consecutively into the reaction vial. After incubation for $120 \mathrm{~min}$ utes at room temperature, the reaction progress was determined by iTLC using a $20 \mathrm{mM}$ citric acid ( $\mathrm{pH}$ 4.9-5.1) mobile phase. The reaction conversion was consistently greater than $90 \% .{ }^{89} \mathrm{Zr}$-labeled $\mathrm{Ab}$ was purified from starting material using a PD-10 column and a gentisic acid mobile phase $(5 \mathrm{mg} / \mathrm{ml}$ of gentisic acid in $0.25 \mathrm{M}$ sodium acetate, $\mathrm{pH}$ 5.4-5.6). The ${ }^{89} \mathrm{Zr}$-labeled $\mathrm{Ab}$ solution was adjusted to $\mathrm{pH} 7.0$ prior to i.v. injection. The purity of the ${ }^{89} \mathrm{Zr}$-labeled Ab was consistently greater than $99 \%$.

Small animal PET/CT and biodistribution studies. Tumor-bearing mice ( $n=4-5$ per treatment arm) received approximately $200 \mu \mathrm{Ci}$ of ${ }^{89} \mathrm{Zr}$-anti-CTLA4 in $100 \mu \mathrm{l}$ volume i.v. using a custom mouse tail vein catheter with a 28 -gauge needle and 100-150 mm-long polyethylene microtubing $(0.28 \mathrm{~mm}$ inner diameter, $0.64 \mathrm{~mm}$ outer diameter, Scientific Commodities Inc.). For control studies, the ${ }^{89} \mathrm{Zr}$-anti-CTLA4 construct was heat denatured in PBS (aq.) for 10 minutes at $95^{\circ} \mathrm{C}$. The mice were imaged on a dedicated small animal PET/CT scanner (Inveon, Siemens Healthcare). At the indicated time points after injection, animals were scanned for 40 minutes for PET and for 10 minutes for CT. The coregistration between PET and CT images was obtained using the rigid transformation matrix from the manufacturer-provided scanner calibration procedure, since the geometry between PET and CT remained constant for each PET/CT scan using the combined PET/ CT scanner. Animals were anesthetized during the imaging study with isoflurane at $2 \%$ concentration mixed with medical grade oxygen.

An ordered subsets expectation maximization (OS-EM) algorithm provided by the manufacturer was used for PET reconstruction. This resulted in $128 \times 128 \times 159$ matrices with a voxel size of $0.776 \times 0.776$ $\times 0.796 \mathrm{~mm}^{3}$. The CT image was created using a conebeam Feldkamp reconstruction algorithm (COBRA) provided by Exxim Computing Corp. The matrix size of the reconstructed CT images was $512 \times 512$ $\times 662$, with an isotropic voxel size of $0.191 \times 0.191 \times 0.191 \mathrm{~mm}^{3}$. The photon attenuation correction was performed for PET reconstruction using the coregistered CT-based attenuation map to ensure the quantitative accuracy of the reconstructed PET data. 
For biodistribution studies, animals were euthanized by $\mathrm{CO}_{2}$ asphyxiation after scans were completed. Tissues, including the tumor, were harvested immediately following sacrifice. Blood was acquired by cardiac puncture immediately after euthanasia. The tissues and blood were weighed and counted using a Wizard3 Gamma Counter (PerkinElmer) to assess ${ }^{89} \mathrm{Zr}$ concentration. Calibration with known amounts of ${ }^{89} \mathrm{Zr}$ was performed to determine the amount of activity in each organ. This activity was then decay corrected, and the percentage of the injected dose per gram (\% ID/g) of tissue was calculated and reported.

Statistics. Data shown in this manuscript are presented as mean \pm SEM. Tumor growth curves at different time points were plotted using Prism 7 and analyzed by 2-way ANOVA with a Tukey's post hoc test comparison between groups. Flow cytometry data were analyzed by 1-way ANOVA with a Tukey's post hoc test. A 2-tailed Student's $t$ test, was used as indicated in the figure legends. A $P$ value of less than 0.05 was considered significant.

Study approval. The present studies in animals were reviewed and approved by the IACUC at UCSF.

\section{Author contributions}

CCSP and DS wrote the manuscript, and planned and performed the research. DS, XL, SW, CB, D Banach, D Bowley, FD, JS, WR,
PR, SM, GL, PB, JM, and GK generated Abs, performed experiments, and reviewed the manuscript. JH, CP, AC, and J. Wang performed experiments and measured tumors. MC advised histology and pathology experiments. ME, J. Wei, and YW performed image experiments. GK and LF supervised and planned the research and reviewed the manuscript.

\section{Acknowledgments}

We thank the UCSF Flow Cytometry Core for technical help and the NIH Tetramer Core for generation of the Spas-1 tetramer. This work has been supported by National Cancer Institute (NCI) grants R01CA163012 and R01CA194511 and a Prostate Cancer Foundation Challenge Grant. This study was also supported by research funding from AbbVie.

Address correspondence to: Lawrence Fong, University of California San Francisco, 513 Parnassus Avenue, Room HSE301A, Box 0519, San Francisco, California 94143-0519, USA. Phone: 415.514.9301; Email: lawrence.fong@medicine.ucsf.edu. Or to: Gillian Kingsbury, Agios Pharmaceuticals, 88 Sidney Street, Cambridge, Massachusetts 02139, USA. Phone: 617.649.2025; Email: Gillian.Kingsbury@agios.com.
1. Grosso JF, Jure-Kunkel MN. CTLA-4 blockade in tumor models: an overview of preclinical and translational research. Cancer Immun. 2013;13:5.

2. Curran MA, Montalvo W, Yagita H, Allison JP. PD- 1 and CTLA- 4 combination blockade expands infiltrating $T$ cells and reduces regulatory $\mathrm{T}$ and myeloid cells within $\mathrm{B} 16$ melanoma tumors. Proc Natl Acad Sci U S A. 2010;107(9):4275-4280.

3. Larkin J, Hodi FS, Wolchok JD. Combined nivolumab and ipilimumab or monotherapy in untreated melanoma. $\mathrm{NEngl} \mathrm{J} \mathrm{Med}$. 2015;373(13):1270-1271.

4. Brahmer JR, et al. Management of immunerelated adverse events in patients treated with immune checkpoint inhibitor therapy: American Society of Clinical Oncology Clinical Practice Guideline. JClin Oncol. 2018;36(17):1714-1768.

5. Wolchok JD, et al. Overall survival with combined nivolumab and ipilimumab in advanced melanoma. N Engl JMed. 2017;377(14):1345-1356.

6. Walunas TL, et al. CTLA-4 can function as a negative regulator of $\mathrm{T}$ cell activation. Immunity. 1994;1(5):405-413.

7. Paterson AM, Sharpe AH. Taming tissue-specific T cells: CTLA- 4 reins in self-reactive T cells. Nat Immunol. 2010;11(2):109-111.

8. Ise $\mathrm{W}$, et al. CTLA-4 suppresses the pathogenicity of self antigen-specific $\mathrm{T}$ cells by cell-intrinsic and cell-extrinsic mechanisms. Nat Immunol. 2010;11(2):129-135.

9. Takahashi $\mathrm{T}$, et al. Immunologic self-tolerance maintained by CD25(+)CD4(+) regulatory $\mathrm{T}$ cells constitutively expressing cytotoxic $\mathrm{T}$ lymphocyte-associated antigen 4.JExp Med. 2000;192(2):303-310.

10. Simpson TR, et al. Fc-dependent depletion of tumor-infiltrating regulatory $\mathrm{T}$ cells co-defines the efficacy of anti-CTLA-4 therapy against melanoma. J Exp Med. 2013;210(9):1695-1710.
11. Cha $\mathrm{E}$, et al. Improved survival with $\mathrm{T}$ cell clonotype stability after anti-CTLA- 4 treatment in cancer patients. Sci Transl Med. 2014;6(238):238ra70.

12. Spain L, Diem S, Larkin J. Management of toxicities of immune checkpoint inhibitors. Cancer Treat Rev. 2016;44:51-60.

13. Ishihara J, et al. Matrix-binding checkpoint immunotherapies enhance antitumor efficacy and reduce adverse events. Sci Transl Med. 2017;9(415):eaan0401.

14. Fassò M, et al. SPAS-1 (stimulator of prostatic adenocarcinoma-specific T cells)/SH3GLB2: A prostate tumor antigen identified by CTLA-4 blockade. Proc Natl Acad Sci U S A. 2008;105(9):3509-3514.

15. Bok RA, Hansell EJ, Nguyen TP, Greenberg NM, McKerrow JH, Shuman MA. Patterns of protease production during prostate cancer progression: proteomic evidence for cascades in a transgenic model. Prostate Cancer Prostatic Dis. 2003;6(4):272-280.

16. Truillet C, et al. Imaging PD-L1 Expression with ImmunoPET. Bioconjug Chem. 2018;29(1):96-103.

17. Fecher LA, Agarwala SS, Hodi FS, Weber JS. Ipilimumab and its toxicities: a multidisciplinary approach. Oncologist. 2013;18(6):733-743.

18. Hodi FS, et al. Improved survival with ipilimumab in patients with metastatic melanoma. $N$ Engl J Med. 2010;363(8):711-723.

19. Robert C, et al. Ipilimumab plus dacarbazine for previously untreated metastatic melanoma. N Engl J Med. 2011;364(26):2517-2526.

20. Kleiner DE, Berman D. Pathologic changes in ipilimumab-related hepatitis in patients with metastatic melanoma. Dig Dis Sci. 2012;57(8):2233-2240.

21. Barjaktarevic IZ, Qadir N, Suri A, Santamauro JT, Stover D. Organizing pneumonia as a side effect of ipilimumab treatment of melanoma. Chest.
2013;143(3):858-861

22. Franzen D, et al. Ipilimumab and early signs of pulmonary toxicity in patients with metastastic melanoma: a prospective observational study. Cancer Immunol Immunother. 2018;67(1):127-134.

23. Illouz F, et al. Endocrine toxicity of immune checkpoint inhibitors: essential crosstalk between endocrinologists and oncologists. Cancer Med. 2017;6(8):1923-1929.

24. Sznol M, et al. Endocrine-related adverse events associated with immune checkpoint blockade and expert insights on their management. Cancer Treat Rev. 2017;58:70-76.

25. Peggs KS, Quezada SA, Chambers CA, Korman AJ, Allison JP. Blockade of CTLA-4 on both effector and regulatory $\mathrm{T}$ cell compartments contributes to the antitumor activity of anti-CTLA-4 antibodies. J Exp Med. 2009;206(8):1717-1725.

26. Komatsu N, Hori S. Full restoration of peripheral Foxp3+ regulatory $\mathrm{T}$ cell pool by radioresistant host cells in scurfy bone marrow chimeras. Proc Natl Acad Sci U S A. 2007;104(21):8959-8964.

27. Ostanin DV, et al. T cell transfer model of chronic colitis: concepts, considerations, and tricks of the trade. Am J Physiol Gastrointest Liver Physiol. 2009;296(2):G135-G146.

28. Morrissey PJ, Charrier K, Braddy S, Liggitt D, Watson JD. CD4+ T cells that express high levels of CD45RB induce wasting disease when transferred into congenic severe combined immunodeficient mice. Disease development is prevented by cotransfer of purified CD4+ T cells. JExp Med.1993;178(1):237-244.

29. Izcue A, Coombes JL, Powrie F. Regulatory T cells suppress systemic and mucosal immune activation to control intestinal inflammation. Immunol Rev. 2006;212:256-271.

30. Liakou CI, et al. CTLA-4 blockade increases IFNgamma-producing CD4+ICOShi cells to shift the ratio of effector to regulatory $\mathrm{T}$ cells 
in cancer patients. Proc Natl Acad Sci U S A. 2008;105(39):14987-14992.

31. Tivol EA, Borriello F, Schweitzer AN, Lynch WP, Bluestone JA, Sharpe AH. Loss of CTLA-4 leads to massive lymphoproliferation and fatal multiorgan tissue destruction, revealing a critical negative regulatory role of CTLA-4. Immunity. 1995;3(5):541-547.

32. Michot JM, et al. Immune-related adverse events with immune checkpoint blockade: a comprehensive review. Eur J Cancer. 2016;54:139-148.

33. Tai X, et al. Basis of CTLA-4 function in regulatory and conventional CD4(+) T cells. Blood. 2012;119(22):5155-5163.

34. Sandin LC, Eriksson F, Ellmark P, Loskog AS, Tötterman TH, Mangsbo SM. Local CTLA4 blockade effectively restrains experimental pancreatic adenocarcinoma growth in vivo. Oncoimmunology. 2014;3(1):e27614.

35. Tang Q, Bluestone JA. The Foxp3+ regulatory $\mathrm{T}$ cell: a jack of all trades, master of regulation. Nat Immunol. 2008;9(3):239-244.

36. Leach DR, Krummel MF, Allison JP. Enhancement of antitumor immunity by CTLA-4 blockade. Science. 1996;271(5256):1734-1736.

37. Klocke K, Sakaguchi S, Holmdahl R, Wing K.
Induction of autoimmune disease by deletion of CTLA-4 in mice in adulthood. Proc Natl Acad Sci US A. 2016;113(17):E2383-E2392.

38. Saffran DC, Raitano AB, Hubert RS, Witte ON, Reiter RE, Jakobovits A. Anti-PSCA mAbs inhibit tumor growth and metastasis formation and prolong the survival of mice bearing human prostate cancer xenografts. Proc Natl Acad Sci U S A. 2001;98(5):2658-2663.

39. Beers SA, Glennie MJ, White AL. Influence of immunoglobulin isotype on therapeutic antibody function. Blood. 2016;127(9):1097-1101.

40. Dahan R, Sega E, Engelhardt J, Selby M, Korman AJ, Ravetch JV. Fc $\gamma R$ s modulate the anti-tumor activity of antibodies targeting the PD-1/PD-L1 axis. Cancer Cell. 2015;28(3):285-295.

41. Romano E, et al. Ipilimumab-dependent cellmediated cytotoxicity of regulatory $\mathrm{T}$ cells ex vivo by nonclassical monocytes in melanoma patients. Proc Natl Acad Sci U S A. 2015;112(19):6140-6145.

42. Kavanagh B, et al. CTLA4 blockade expands FoxP3+ regulatory and activated effector CD4+ $\mathrm{T}$ cells in a dose-dependent fashion. Blood. 2008;112(4):1175-1183.

43. Sharma A, et al. Anti-CTLA-4 immunotherapy does not deplete FOXP3+ regulatory T cells
(Tregs) in human cancers [published online ahead of print July 27, 2018]. Clin Cancer Res. https://doi. org/10.1158/1078-0432.CCR-18-0762.

44. Selby MJ, et al. Anti-CTLA-4 antibodies of IgG2a isotype enhance antitumor activity through reduction of intratumoral regulatory $\mathrm{T}$ cells. Cancer Immunol Res. 2013;1(1):32-42.

45. Smith P, DiLillo DJ, Bournazos S, Li F, Ravetch JV. Mouse model recapitulating human Fcy receptor structural and functional diversity. Proc Natl Acad Sci U S A. 2012;109(16):6181-6186.

46. Arce Vargas F, et al. Fc effector function contributes to the activity of human anti-ctla- 4 antibodies. Cancer Cell. 2018;33(4):649-663.e4.

47. Gül N, van Egmond M. Antibody-dependent phagocytosis of tumor cells by macrophages: a potent effector mechanism of monoclonal antibody therapy of cancer. Cancer Res. 2015;75(23):5008-5013.

48. Wu C, et al. Simultaneous targeting of multiple disease mediators by a dual-variabledomain immunoglobulin. Nat Biotechnol. 2007;25(11):1290-1297.

49. Gu J, Ghayur T. Generation of dual-variable-domain immunoglobulin molecules for dual-specific targeting. Meth Enzymol. 2012;502:25-41. 